\title{
CONCERNING THE WAVE EQUATION ON ASYMPTOTICALLY EUCLIDEAN MANIFOLDS
}

\author{
CHRISTOPHER D. SOGGE AND CHENGBO WANG
}

\begin{abstract}
We obtain KSS, Strichartz and certain weighted Strichartz estimates for the wave equation on $\left(\mathbb{R}^{d}, \mathfrak{g}\right), d \geq 3$, when metric $\mathfrak{g}$ is non-trapping and approaches the Euclidean metric like $\langle x\rangle^{-\rho}$ with $\rho>0$. Using the KSS estimate, we prove almost global existence for quadratically semilinear wave equations with small initial data for $\rho>1$ and $d=3$. Also, we establish the Strauss conjecture when the metric is radial with $\rho>0$ for $d=3$.
\end{abstract}

\section{Contents}

1. Introduction and Main Results

2. KSS Estimates

3. Higher Order KSS Estimates 10

4. Almost Global Existence $\quad 12$

5. Global Strichartz Estimates 14

6. Weighted Strichartz Estimates 18

7. Strauss Conjecture 22

References $\quad 24$

\section{Introduction and Main Results}

This paper is devoted to the study of the semilinear wave equation on asymptotically Euclidean non-trapping Riemannian manifolds. We shall obtain almost global existence for quadratic semilinear wave equations with small data, and show that the Strauss conjecture holds in this setting, in dimension $d=3$.

In Minkowski space, the quadratically semilinear wave equation has been thoroughly studied. Global existence is known in dimension $d \geq 4$ for small initial data (see Klainerman and Ponce [19] and references therein). Almost global existence in dimension $d=3$ for small data was shown by John and Klainerman in [11. Almost global means that the life time of a solution is at least $\exp (c / \delta)$ with some $c>0$, where $\delta$ is the size of the initial data in some appropriate Sobolev space. Note that, in dimension $d=3$, Sideris [27] has proved that global existence does not hold in general (see also John [10]).

The first author was supported by the National Science Foundation. The second author was supported in part by NSFC 10871175 . 
In [14, Keel, Smith and Sogge gave a new proof of the almost global existence result in dimension 3 using estimates (known as KSS estimates) of the form

$$
(\ln (2+T))^{-1 / 2}\left\|\langle x\rangle^{-1 / 2} u^{\prime}\right\|_{L^{2}\left([0, T] \times \mathbb{R}^{3}\right)} \lesssim\left\|u^{\prime}(0, \cdot)\right\|_{L^{2}\left(\mathbb{R}^{3}\right)}+\int_{0}^{T}\|F(s, \cdot)\|_{L^{2}\left(\mathbb{R}^{3}\right)} d s,
$$

and a certain Sobolev type estimate due to Klainerman (see [18]). Here $u$ solves the wave equation $\square u=F$ in $[0,+\infty) \times \mathbb{R}^{d}$ and $u^{\prime}=\left(\partial_{t} u, \partial_{x} u\right)$. Earlier versions of (1.1) appeared before. The first ones appear to be due to Morawetz [26] and Strauss [33, who proved somewhat weaker versions of (1.1). See also [17. In [14] existence results for the non-trapping obstacle case were also obtained. In [15, similar results were obtained for the corresponding quasilinear equation (see also Metcalfe-Sogge [23]).

Recently, Bony and Häfner 3 obtained a weaker version of KSS estimate in the current setting and proved the long time existence for quadratic semilinear wave equations with small data. In the present paper, by using results of MetcalfeSogge [23, we are able to prove the full KSS estimate, and hence the almost global existence for the quadratic semilinear wave equation.

Recently, in Minkowski space, Fang and Wang [6] and Hidano-Metcalfe-SmithSogge-Zhou [9] proved the Strauss conjecture with low regularity for $d=2,3,4$, by using a weighted Strichartz estimate of the form

$$
\left\||x|^{\frac{n}{2}-\frac{n+1}{r}-\gamma} u\right\|_{L_{t,|x|}^{r} L_{\omega}^{2}} \lesssim\|u(0, \cdot)\|_{\dot{H}_{x}^{\gamma}}+\left\|\partial_{t} u(0, \cdot)\right\|_{\dot{H}_{x}^{\gamma-1}}+\|F\|_{L_{t}^{1} \dot{H}_{x}^{\gamma-1}}
$$

for $\gamma \in(1 / 2-1 / r, d / 2-1 / r), r \in[2, \infty]$, where we use the notation

$$
\|f\|_{L_{t}^{q} L_{|x|}^{r} L_{\omega}^{p}}=\left\|\left(\int_{0}^{\infty}\|f(t,|x| \omega)\|_{L_{\omega}^{p}}^{r}|x|^{d-1} d|x|\right)^{1 / r}\right\|_{L_{t}^{q}}
$$

with $L_{\omega}^{p}$ denoting the $L^{p}$-norm on $S^{n-1}$ with respect to the standard measure. In this paper, we obtain a somewhat weaker version of this estimate in this general setting, which suffices for us to establish the Strauss conjecture for $d=3$ (when the metric is radial).

Using ideas from Burq 4], Metcalfe [22, Smith-Sogge [30] and Hidano-MetcalfeSmith-Sogge-Zhou [9, we can also use the local energy decay estimates to prove global Strichartz estimates in this setting. We should point out, though, that the idea that, in many situations, local energy estimates can be used to prove global Strichartz estimates occurs in many other works. The first seems to be that of Journé, Soffer and Sogge 12 who proved global Strichartz estimates for Schrödinger operators with potential using local energy estimates (local smoothing) for $e^{i t \Delta}$. Staffilani and Tataru 32 extended this philosphy by considering more general perturbations of $\Delta$, and more recently Metcalfe and Tataru 24] used the philosophy that local energy estimates imply Strichartz estimates to handle (small) metric perturbations of $\square=\partial_{t}^{2}-\Delta$. Thus, in many ways, some of the techniques employed in this paper are not novel, since they have been used in many earlier works. A slight novelty, though, might be that we obtain our global estimates by combining local energy estimates (in this case due to Bony and Häfner [3]) with global Strichartz estimates not involving $\Delta$, but rather small perturbations of the Laplacian. The ones that allow us to prove the aforementioned (sharp) KSS estimates are due to Metcalfe and the first author 23, while the ones that allow 
us to prove the standard mixed-norm Strichartz estimates are due to Metcalfe and Tataru [24].

Let us now state our precise results. We consider asymptotically Euclidean manifolds $\left(\mathbb{R}^{d}, \mathfrak{g}\right)$ with $d \geq 3$ and

$$
\mathfrak{g}=\sum_{i, j=1}^{d} g_{i j}(x) d x^{i} d x^{j} .
$$

We suppose $g_{i j}(x) \in C^{\infty}\left(\mathbb{R}^{d}\right)$ and, for some $\rho>0$,

$$
\forall \alpha \in \mathbb{N}^{d} \quad \partial_{x}^{\alpha}\left(g_{i j}-\delta_{i j}\right)=\mathcal{O}\left(\langle x\rangle^{-|\alpha|-\rho}\right),
$$

with $\delta_{i j}=\delta^{i j}$ being the Kronecker delta function. We also assume that

$$
\mathfrak{g} \text { is non-trapping. }
$$

Let $g(x)=(\operatorname{det}(\mathfrak{g}))^{1 / 4}$. The Laplace-Beltrami operator associated with $\mathfrak{g}$ is given by

$$
\Delta_{\mathfrak{g}}=\sum_{i j} \frac{1}{g^{2}} \partial_{i} g^{i j} g^{2} \partial_{j}
$$

where $g^{i j}(x)$ denotes the inverse metric. Note $-\Delta_{\mathfrak{g}}$ is self-adjoint non-negative on $L^{2}\left(\mathbb{R}^{d}, g^{2} d x\right)$, while $P=-g \Delta_{\mathfrak{g}} g^{-1}$ is self-adjoint non-negative on $L^{2}\left(\mathbb{R}^{d}, d x\right)$. Let $\Omega=\Omega_{k, \ell}:=x_{k} \partial_{\ell}-x_{\ell} \partial_{k}$ be the rotational vector fields. We consider the following semilinear wave equation

$$
\left\{\begin{array}{l}
\square_{\mathfrak{g}} u=Q\left(u^{\prime}\right), \quad(t, x) \in \mathbb{R}_{+} \times \mathbb{R}^{d} \\
u(0, x)=u_{0}(x), \quad \partial_{t} u(0, x)=u_{1}(x), \quad x \in \mathbb{R}^{d} .
\end{array}\right.
$$

Here $\square_{\mathfrak{g}}=\partial_{t}^{2}-\Delta_{\mathfrak{g}}$ and $Q\left(u^{\prime}\right)$ is a quadratic form in $u^{\prime}=\left(\partial_{t} u, \partial_{x} u\right)$. One of our main results is the following theorem.

Theorem 1.1. Assume hypotheses ([H1) and (H2) with $\rho>1$. Suppose $u_{0}, u_{1} \in$ $C_{0}^{\infty}\left(\mathbb{R}^{3}\right)$, and

$$
\sum_{|\alpha|+|\beta| \leq 5}\left\|\partial_{x}^{\beta} \Omega^{\alpha} u_{0}\right\|_{L_{x}^{2}}+\sum_{|\alpha|+|\beta| \leq 4}\left\|\partial_{x}^{\beta} \Omega^{\alpha} u_{1}\right\|_{L_{x}^{2}} \leq \delta .
$$

For $\delta$ small enough, the problem (1.3) has a unique almost global solution $u \in$ $C^{\infty}\left(\left[0, T_{\delta}\right] \times \mathbb{R}^{d}\right)$ with $T_{\delta}=\exp (c / \delta)$ for some $c>0$.

The main ingredient of the proof are estimates of type (1.1). Let us therefore consider the corresponding linear equation. Let $u$ be solution of

$$
\left\{\begin{array}{l}
\left(\partial_{t}^{2}-\Delta_{\mathfrak{g}}\right) u(t, x)=F(t, x), \quad(t, x) \in \mathbb{R}_{+} \times \mathbb{R}^{d} \\
u(0, x)=u_{0}(x), \quad \partial_{t} u(0, x)=u_{1}(x), \quad x \in \mathbb{R}^{d} .
\end{array}\right.
$$

With the notation

$$
A_{\mu}(T)= \begin{cases}\ln (2+T)^{-1 / 2} & \mu=1 / 2, \\ 1 & \mu>1 / 2,\end{cases}
$$

we have the following KSS estimate. 
Theorem 1.2. Assume that (H1) and (H2) hold with $\rho>0$ and let $\mu \geq 1 / 2$, $d \geq 3$. For all $\varepsilon>0$, the solution of (1.5) satisfies

$$
A_{\mu}(T)\left(\left\|\langle x\rangle^{-\mu} u^{\prime}\right\|_{L_{T}^{2} L_{x}^{2}}+\left\|\langle x\rangle^{-\mu-1} u\right\|_{L_{T}^{2} L_{x}^{2}}\right) \lesssim\left\|u^{\prime}(0, \cdot)\right\|_{L_{x}^{2}}+\left\|\langle x\rangle^{\frac{1}{2}+\varepsilon} F\right\|_{L_{T}^{2} L_{x}^{2}},
$$

where we use $L_{T}^{2}$ to denote $L_{t \in[0, T]}^{2}$.

We remark that our estimate (1.6) agrees with ones in Bony and Häfner 3 when $\mu>1 / 2$, while for $\mu=1 / 2$ they are slightly stronger since we obtain the sharp bounds with $A_{1 / 2}(T)=(\ln (2+T))^{-1 / 2}$, as opposed to the bounds of $T^{-\varepsilon}, \varepsilon>0$, in [3. This improvement allows us to obtain the almost global existence results alluded to before. On the other hand, our proof is very similar to that of Bony and Häfner [3] and papers that preceded it, starting with [14]. A slight point of departure is that we combine local energy estimates (due to Bony and Häfner [3]) not with global KSS estimates for $\Delta$ but rather for small metric perturbations of $\Delta$ (which are due to Metcalfe and the first author [23]).

To prove the nonlinear theorem, we need to get higher order estimates. For this purpose, let us put $Z=\left\{\partial_{t}, \partial_{x}, \Omega\right\}, Y=\left\{\partial_{x}, \Omega\right\}, X=\left\{\partial_{x}\right\}$. Then, we have

Theorem 1.3. Assume that (H1) and (H2) hold with $\rho>1$. Let $N \geq 0$ and $\mu \geq 1 / 2$. The solution of (1.5) satisfies

$$
\begin{aligned}
\sup _{0 \leq t \leq T} \sum_{1 \leq k+j \leq N+1}\left\|\partial_{t}^{k} P^{j / 2} g u(t, \cdot)\right\|_{L_{x}^{2}}+\sum_{|\alpha| \leq N} A_{\mu}(T)\left\|\langle x\rangle^{-\mu}\left(\left|\left(Z^{\alpha} u\right)^{\prime}\right|+\frac{\left|Z^{\alpha} u\right|}{\langle x\rangle}\right)\right\|_{L_{T}^{2} L_{x}^{2}} \\
\qquad \sum_{|\alpha| \leq N}\left\|\left(Y^{\alpha} u\right)^{\prime}(0, \cdot)\right\|_{L_{x}^{2}}+\sum_{|\alpha| \leq N} \int_{0}^{T}\left\|Z^{\alpha} F(s, \cdot)\right\|_{L_{x}^{2}} d s .
\end{aligned}
$$

Note that the estimate (1.6) can be viewed as the local energy decay estimate for $\square_{\mathfrak{g}}$. The local Strichartz estimates for variable coefficient wave equations have been studied extensively, see e.g. Kapitanski [13, Mockenhaupt-Seeger-Sogge [25, Smith 29], Bahouri-Chemin [1] 22, Tataru 34] 35] 37]. And recently, Metcalfe and Tataru have obtained global Strichartz estimates involving small perturbations of the Minkowski metric in [24]. As we mentioned before, by combining these with local energy estimates we shall prove global Strichartz estimates for $\square_{\mathfrak{g}}$.

For the Minkowski case, it is known ([16, 20]) that we have Strichartz estimates if $(s, q, r)$ is admissible, i.e.,

$$
\frac{1}{q} \leq \min \left(\frac{1}{2}, \frac{d-1}{2}\left(\frac{1}{2}-\frac{1}{r}\right)\right), \quad(q, r) \neq(2, \infty),(\infty, \infty), \quad s=d\left(\frac{1}{2}-\frac{1}{r}\right)-\frac{1}{q} .
$$

Our global Strichartz estimates for $\square_{\mathfrak{g}}$ are the following.

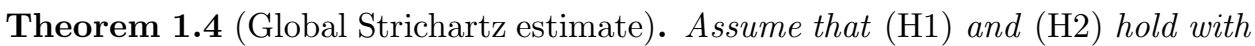
$\rho>0, d \geq 3$ and $s \in[0,1](s \in(0,1)$ if $d=3)$. The solution of (1.5) satisfies

$$
\|u\|_{L_{t}^{q} L_{x}^{r}} \lesssim\left\|u_{0}\right\|_{\dot{H}^{s}}+\left\|u_{1}\right\|_{\dot{H}^{s-1}}+\|F\|_{L_{t}^{1} \dot{H}^{s-1}},
$$

for any admissible $(s, q, r)$ with $q>2, r<\infty$.

The proofs of Theorem 1.2 and Theorem 1.4 follow a similar strategy. We first use results from [23] and 24] to show that we can construct a metric $\tilde{\mathfrak{g}}$ which agrees with $\mathfrak{g}$ near infinity and has the property that the bounds in these two theorems are valid if $\square_{\mathfrak{g}}$ is replaced by $\square_{\mathfrak{g}}$. Then, by adapting arguments from [4] and [30], 
we can use these estimates along with the local energy decay estimates for $\square_{\mathfrak{g}}$ (see Lemma 2.6 below) to show that $\square_{\mathfrak{g}}$ satisfies the same global estimates as its compact perturbation $\square_{\tilde{\mathfrak{g}}}$.

Now let us describe the weighted Strichartz estimate and its application to Strauss conjecture in this general setting. Let $p>1$,

$$
s_{c}=\frac{d}{2}-\frac{2}{p-1}, \quad s_{s b}=\frac{1}{2}-\frac{1}{p} .
$$

The equation that we shall consider is

$$
\left\{\begin{array}{l}
\left(\partial_{t}^{2}-\Delta_{\mathfrak{g}}\right) u(t, x)=F_{p}(u(t, x)), \quad(t, x) \in \mathbb{R}_{+} \times \mathbb{R}^{d} \\
u(0, x)=u_{0}(x), \quad \partial_{t} u(0, x)=u_{1}(x), \quad x \in \mathbb{R}^{d},
\end{array}\right.
$$

We shall assume that the nonlinear term behaves like $|u|^{p}$, and so we assume that

$$
\sum_{0 \leq j \leq 1}|u|^{j}\left|\partial_{u}^{j} F_{p}(u)\right| \lesssim|u|^{p}
$$

See [31], $§ 4.4$ for a discussion about how $s \geq s_{s b}$ is needed for local existence, while $s_{c}$ is critical for global existence.

We can now state our existence theorem for (1.9). Due to some technical difficulties, we are only able to deal with only the case where $g^{i j}(x)=h(|x|) \delta^{i j}$ for some function $h$.

Theorem 1.5. Assume that $g^{i j}(x)=h(|x|) \delta^{i j}$ for some function $h$, (H1) and (स्H2) hold with $\rho>0, d=3$ and $p>p_{c}=1+\sqrt{2}$. Then for any $\epsilon>0$ such that

$$
s=s_{c}-\epsilon \in\left(s_{s b}, 1 / 2\right),
$$

there is an $\delta>0$ depending on $p$ so that (1.9) has a global solution satisfying $\left(Y^{\alpha} u(t, \cdot), \partial_{t} Y^{\alpha} u(t, \cdot)\right) \in \dot{H}^{s} \times \dot{H}^{s-1},|\alpha| \leq 1, t \in \mathbb{R}_{+}$, whenever the initial data satisfies

$$
\sum_{|\alpha| \leq 1}\left(\left\|Y^{\alpha} u_{0}\right\|_{\dot{H}^{s}}+\left\|Y^{\alpha} u_{1}\right\|_{\dot{H}^{s-1}}\right)<\delta
$$

with $0<\delta<\delta_{0}$.

Existence results of this type when $\Delta_{g}=\Delta$ are a celebrated result of John [10]. Subsequently, Strauss conjectured that for dimensions $d \geq 2$ the critical exponent for small data global existence for equations of the form (1.9) (when $\Delta_{g}=\Delta$ ) should be the positive root of the equation $(d-1) p^{2}-(d+1) p-2=0$. This conjecture was settled for the Minkowski space case in 7], [8, [21, [28, and [38]. See [31] for further discussion.

As in the case of (1.3), the main ingredient of the proof are estimates of type (1.2). If we consider the corresponding linear equation (1.5), then we have the following estimate, where the metric is not restricted to the special case where $g^{i j}(x)=h(|x|) \delta^{i j}$.

Theorem 1.6. Assume that (H1) and (H2) hold with $\rho>0, d \geq 3,2<q \leq \infty$ and $s \in\left(s_{s b}(q), 1\right]$. For all $\varepsilon, \eta>0$ small enough, the solution of (1.5) satisfies (1.13)

$$
\left\||x|^{\frac{d}{2}-\frac{d+1}{q}-s-\varepsilon} u\right\|_{L_{t,|x| \geq R}^{q} L_{\omega}^{2+\eta}}+\left\|\langle x\rangle^{-\frac{1}{2}-s-\varepsilon} u\right\|_{L_{t, x}^{2}} \lesssim\left\|u_{0}\right\|_{\dot{H}_{x}^{s}}+\left\|u_{1}\right\|_{\dot{H}_{x}^{s-1}}+\|F\|_{L_{t}^{1} \dot{H}_{x}^{s-1}}
$$


We should comment on the hypotheses in the existence theorems. First, because of the various commutator terms that arise in the proofs we are, at present, only able to handle semilinear terms in the existence theorems involving quadratic nonlinearities, as opposed the the quasilinear case (for $\square_{g}=\partial_{t}^{2}-\Delta$ ) treated in [15. For similar reasons, in our results involving the Strauss conjecture, due to difficulties in dealing with commutators involving the $\Omega$ vector fields and $\square_{\mathfrak{g}}$ we have to assume that the metric $\mathfrak{g}$ is spherically symmetric. For similar reasons, although the linear estimates just require the hypothesis that $\rho>0$ currently our techniques require the assumption that $\rho>1$ in the hypotheses of some of the nonlinear theorems. We do not know, however, what the natural assumption regarding $\rho$ should be for the latter, though.

\section{KSS Estimates}

In this section, we give the proof of the KSS estimates. First, we will need the following lemmas, where we denote $\widetilde{\partial}_{x}:=\partial_{x} g^{-1}$.

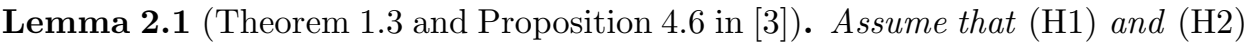
hold with $\rho>0$, then for all $\epsilon>0$, the solution of the equation $\left(\partial_{t}^{2}+P\right) u=F$ satisfies

$$
\left\|\langle x\rangle^{-\frac{1}{2}-\epsilon}\left(\partial_{t}, P^{1 / 2}\right) u\right\|_{L^{2}\left(\mathbb{R} \times \mathbb{R}^{d}\right)} \lesssim\left\|\left(\partial_{t}, P^{1 / 2}\right) u(0, \cdot)\right\|_{L^{2}\left(\mathbb{R}^{d}\right)}+\left\|\langle x\rangle^{\frac{1}{2}+\epsilon} F\right\|_{L^{2}\left(\mathbb{R} \times \mathbb{R}^{d}\right)} .
$$

Remark 2.1. In fact, from the proof of Proposition 4.4 and 4.6 in [3], we also have

$$
\left\|\langle x\rangle^{-\frac{3}{2}-\epsilon} u\right\|_{L^{2}\left(\mathbb{R} \times \mathbb{R}^{d}\right)} \lesssim\left\|\left(\partial_{t}, P^{1 / 2}\right) u(0, \cdot)\right\|_{L^{2}\left(\mathbb{R}^{d}\right)}+\left\|\langle x\rangle^{\frac{1}{2}+\epsilon} F\right\|_{L^{2}\left(\mathbb{R} \times \mathbb{R}^{d}\right)} .
$$

Lemma 2.2 (Theorem 5.1 in [23]). Let $\square_{h}=\partial_{t}^{2}-\Delta+h^{\alpha \beta}(t, x) \partial_{\alpha} \partial_{\beta}, h^{\alpha \beta}=h^{\beta \alpha}$ and $\sum\left|h^{\alpha \beta}\right| \leq \delta$. Then if $\delta>0$ is small enough, $d \geq 3$, the solution to the equation $\square_{h} u=F$ satisfies

$(\ln (2+T))^{-1}\left\|\langle x\rangle^{-1 / 2}\left(\left|u^{\prime}\right|+\frac{|u|}{\langle x\rangle}\right)\right\|_{L^{2}\left([0, T] \times \mathbb{R}^{d}\right)}^{2}+\left\|\langle x\rangle^{-1 / 2-\epsilon}\left(\left|u^{\prime}\right|+\frac{|u|}{\langle x\rangle}\right)\right\|_{L^{2}\left([0, T] \times \mathbb{R}^{d}\right)}^{2}$

$$
\lesssim\left\|u^{\prime}(0, \cdot)\right\|_{L^{2}\left(\mathbb{R}^{d}\right)}^{2}+\int_{0}^{T} \int\left(\left|u^{\prime}\right|+\frac{u}{|x|}\right)\left(|F|+\left(\left|h^{\prime}\right|+\frac{h}{|x|}\right)\left|u^{\prime}\right|\right) d x d t
$$

for any $\epsilon>0$.

To obtain higher order estimates, we will need the following.

Lemma 2.3 (Lemma B.13, 4.1 and 4.2 in [3]). For all $-3 / 2 \leq \tilde{\mu}<\mu \leq 3 / 2$, we have

$$
\begin{gathered}
\left\|\langle x\rangle^{-\mu} \widetilde{\partial}_{\ell} u\right\|_{L^{2}\left(\mathbb{R}^{d}\right)} \lesssim\left\|\langle x\rangle^{-\widetilde{\mu}} P^{1 / 2} u\right\|_{L^{2}\left(\mathbb{R}^{d}\right)}, \\
\left\|\langle x\rangle^{-\mu} P^{1 / 2} u\right\|_{L^{2}\left(\mathbb{R}^{d}\right)} \lesssim \sum_{\ell=1}^{d}\left\|\langle x\rangle^{-\widetilde{\mu}} \widetilde{\partial}_{\ell} u\right\|_{L^{2}\left(\mathbb{R}^{d}\right)} .
\end{gathered}
$$

Also, for $u \in H^{1}\left(\mathbb{R}^{d}\right)$,

$$
\left\|P^{1 / 2} u\right\|_{L^{2}\left(\mathbb{R}^{d}\right)} \lesssim\left\|\nabla g^{-1} u\right\|_{L^{2}\left(\mathbb{R}^{d}\right)} \lesssim\left\|P^{1 / 2} u\right\|_{L^{2}\left(\mathbb{R}^{d}\right)} .
$$


We also need a lemma which says that the homogeneous spaces defined by $P$ and $-\Delta$ are essentially the same. In what follows, "remainder terms", $r_{j}, j \in \mathbb{N}$, will denote a smooth function such that

$$
\partial_{x}^{\alpha} r_{j}(x)=\mathcal{O}\left(\langle x\rangle^{-\rho-j-|\alpha|}\right)
$$

Lemma 2.4. If $s \in[-1,1]$, then

$$
\|u\|_{\dot{H}^{s}} \simeq\left\|P^{s / 2} u\right\|_{L_{x}^{2}} .
$$

If $s \in[0,1]$,

$$
\begin{aligned}
\left\|\tilde{\partial}_{j} u\right\|_{\dot{H}^{-s}} & \lesssim\left\|P^{1 / 2} u\right\|_{\dot{H}^{-s}}, \\
\left\|P^{1 / 2} u\right\|_{\dot{H}^{s}} & \lesssim \sum_{j}\left\|\tilde{\partial}_{j} u\right\|_{\dot{H}^{s}} .
\end{aligned}
$$

Moreover, we have for $s \in(0,2]$ and $1<q<d / s$,

$$
\left\|P^{s / 2} u\right\|_{L_{x}^{q} \lesssim\|u\|_{\dot{H}^{s, q}}} .
$$

Proof. For the first estimate (2.8), by interpolation and duality, we need only to prove the estimate for the special case where $s=1$, i.e.

$$
\|\nabla u\|_{L^{2}} \simeq\left\|\nabla g^{-1} u\right\|_{L^{2}} .
$$

In fact,

$$
\left\|\nabla g^{-1} u\right\|_{L^{2}} \lesssim\left\|\left(\nabla g^{-1}\right) u\right\|_{L^{2}}+\left\|g^{-1} \nabla u\right\|_{L^{2}} \lesssim\left(\left\|\nabla g^{-1}\right\|_{L^{d}}+\left\|g^{-1}\right\|_{L^{\infty}}\right)\|\nabla u\|_{L^{2}} .
$$

By the hypotheses (H1) and the ellipticity of $P$, we know that

Image $(g) \subset\left(\delta, \delta^{-1}\right), \quad|\partial g|,\left|\partial g^{-1}\right|=\mathcal{O}\left(\langle x\rangle^{-1-\rho}\right) \in L^{d}, \quad\left|\partial^{2} g^{-1}\right|=\mathcal{O}\left(\langle x\rangle^{-2-\rho}\right) \in L^{d / 2}$, for some $\delta>0$. So we know that

$$
\|\nabla h u\|_{L^{2}} \lesssim\|\nabla u\|_{L^{2}}
$$

whenever $h=g$ or $h=g^{-1}$.

To prove (2.9), we note first that by the first inequality (2.8),

$$
\left\|\tilde{\partial}_{j} f\right\|_{\dot{H}^{-1}}=\left\|\partial_{j} g^{-1} f\right\|_{\dot{H}^{-1}} \lesssim\left\|g^{-1} f\right\|_{L^{2}} \lesssim\|f\|_{L^{2}} \lesssim\left\|P^{1 / 2} f\right\|_{\dot{H}^{-1}} .
$$

Thus the inequality (2.9) follows from interpolation with (2.6).

Note that by (2.9),

$$
\left|\left\langle P^{1 / 2} f, P^{1 / 2} h\right\rangle\right|=|\langle P f, h\rangle|=\left|\left\langle g^{2} g^{i j} \tilde{\partial}_{j} f, \tilde{\partial}_{i} h\right\rangle\right| \lesssim \sum_{j}\left\|\tilde{\partial}_{j} f\right\|_{\dot{H}^{s}}\left\|P^{1 / 2} h\right\|_{\dot{H}^{-s}},
$$

this gives (2.10).

For the last inequality, let $a^{i j}=g^{2} g^{i j}$ and $P_{1}=\partial_{i} a^{i j} \partial_{j}$, then $P g=g^{-1} P_{1}$, $a^{i j} \in L^{\infty}$ and $\partial_{i} a^{i j} \in L^{d}$. Denoting $D=\sqrt{-\Delta}$, then for $1<q<d$

$$
\left\|P_{1} D^{-2} u\right\|_{L^{q}} \lesssim\left\|\left(\partial_{i} a^{i j}\right) \partial_{j} D^{-2} u\right\|_{L^{q}}+\left\|a^{i j} D^{-2} \partial_{i} \partial_{j} u\right\|_{L^{q}} \lesssim\|u\|_{L^{q}} .
$$

Thus if $1<q<d / 2$,

$$
\|P u\|_{L^{q}}=\left\|g^{-1} P_{1} g^{-1} u\right\|_{L^{q}} \lesssim\left\|P_{1} g^{-1} u\right\|_{L^{q}} \lesssim\left\|D^{2} g^{-1} u\right\|_{L^{q}} \lesssim\|u\|_{\dot{H}^{2, q}} .
$$

Consequently, the last inequality in the lemma follows from interpolating with the trivial estimate where $s=0$.

Using this we can obtain analogues of Lemma 2.1 and Remark 2.1 involving $-\Delta_{\mathfrak{g}}$. 


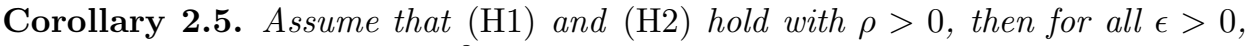
the solution of the equation $\left(\partial_{t}^{2}-\Delta_{\mathfrak{g}}\right) u=F$ satisfies

$$
\left\|\langle x\rangle^{-\frac{1}{2}-\epsilon} \partial_{t, x} u\right\|_{L_{t, x}^{2}}+\left\|\langle x\rangle^{-\frac{3}{2}-\epsilon} u\right\|_{L_{t, x}^{2}} \lesssim\left\|\partial_{t, x} u(0, \cdot)\right\|_{L_{x}^{2}}+\left\|\langle x\rangle^{\frac{1}{2}+\epsilon} F\right\|_{L_{t, x}^{2}} .
$$

Proof. Let $v=g u$ and $G=g F$, then we have

$$
\left(\partial_{t}^{2}-g \Delta_{\mathfrak{g}} g^{-1}\right) v=G
$$

Then by Lemma 2.1, Remark 2.1, (2.4) and (2.6) in Lemma 2.3, we have

$$
\begin{aligned}
\left\|\langle x\rangle^{-\frac{1}{2}-\epsilon} \partial_{t, x} u\right\|_{L_{t, x}^{2}}+\left\|\langle x\rangle^{-\frac{3}{2}-\epsilon} u\right\|_{L_{t, x}^{2}} & \lesssim\left\|\langle x\rangle^{-\frac{1}{2}-\epsilon}\left(\partial_{t}, \tilde{\partial}_{x}\right) v\right\|_{L_{t, x}^{2}}+\left\|\langle x\rangle^{-\frac{3}{2}-\epsilon} v\right\|_{L_{t, x}^{2}} \\
& \lesssim\left\|\langle x\rangle^{-\frac{1}{2}-\epsilon / 2}\left(\partial_{t}, P^{1 / 2}\right) v\right\|_{L_{t, x}^{2}}+\left\|\langle x\rangle^{-\frac{3}{2}-\epsilon / 2} v\right\|_{L_{t, x}^{2}} \\
& \lesssim\left\|\left(\partial_{t}, P^{1 / 2}\right) v(0, \cdot)\right\|_{L_{x}^{2}}+\left\|\langle x\rangle^{\frac{1}{2}+\epsilon / 2} G\right\|_{L_{t, x}^{2}} \\
& \lesssim\left\|\left(\partial_{t}, \tilde{\partial}_{x}\right) v(0, \cdot)\right\|_{L_{x}^{2}}+\left\|\langle x\rangle^{\frac{1}{2}+\epsilon} F\right\|_{L_{t, x}^{2}} \\
& \lesssim\left\|\left(\partial_{t}, \partial_{x}\right) u(0, \cdot)\right\|_{L_{x}^{2}}+\left\|\langle x\rangle^{\frac{1}{2}+\epsilon} F\right\|_{L_{t, x}^{2}} .
\end{aligned}
$$

This completes the proof.

We now establish the local energy decay estimates for the $\square_{\mathfrak{g}}$.

Lemma 2.6 (Local Energy Decay). For the linear equation (1.5), if $F(t, x)=0$ for $|x|>R$ with $R$ fixed, then for fixed $\beta \in C_{0}^{\infty}\left(\mathbb{R}^{d}\right)$, we have

$$
\|\beta u\|_{L_{t}^{2} H^{1}} \lesssim\left\|u_{0}\right\|_{\dot{H}^{1}}+\left\|u_{1}\right\|_{L_{x}^{2}}+\|F\|_{L_{t}^{2} L_{x}^{2}} .
$$

Moreover, if $F \equiv 0$ and $s \in[0,1]$, then

$$
\|\beta u\|_{L_{t}^{2} H^{s}} \lesssim\left\|u_{0}\right\|_{\dot{H}^{s}}+\left\|u_{1}\right\|_{\dot{H}_{x}^{s-1}} .
$$

Proof. By (2.12), noting the support property of the forcing term, we know that

$$
\left\|\langle x\rangle^{-1 / 2-\varepsilon}\left(\partial_{t}, \partial_{x}\right) u\right\|_{L_{t, x}^{2}}+\left\|\langle x\rangle^{-3 / 2-\varepsilon} u\right\|_{L_{t, x}^{2}} \lesssim\left\|u_{0}\right\|_{\dot{H}^{1}}+\left\|u_{1}\right\|_{L_{x}^{2}}+\|F\|_{L_{t, x}^{2}} .
$$

Thus

$$
\|\beta u\|_{L_{t}^{2} H^{1}} \lesssim\left\|\langle x\rangle^{-1 / 2-\varepsilon}\left(u, \partial_{x} u\right)\right\|_{L_{t, x}^{2}} \lesssim\left\|u_{0}\right\|_{\dot{H}^{1}}+\left\|u_{1}\right\|_{L_{x}^{2}}+\|F\|_{L_{t, x}^{2}} .
$$

In the case $F=0$, let $v=g u$, then we have $\left(\partial_{t}^{2}-g \Delta_{\mathfrak{g}} g^{-1}\right) v=0$. Note that $\|g u\|_{\dot{H}^{1}} \lesssim\|u\|_{\dot{H}^{1}}$, this gives $\|g u\|_{\dot{H}^{-1}} \lesssim\|u\|_{\dot{H}^{-1}}$ by duality. Thus we have by (2.1),

$\|\beta u\|_{L_{t, x}^{2}} \lesssim\|\beta v\|_{L_{t, x}^{2}} \lesssim\left\|\langle x\rangle^{-1 / 2-\epsilon} v\right\|_{L_{t, x}^{2}} \lesssim\|v(0, \cdot)\|_{L^{2}}+\left\|\partial_{t} v(0, \cdot)\right\|_{\dot{H}^{-1}} \lesssim\left\|u_{0}\right\|_{L^{2}}+\left\|u_{1}\right\|_{\dot{H}^{-1}}$.

This completes the proof by interpolation with (2.13).

Now we are ready to give the proof of the KSS estimates presented in Theorem 1.2. In the proof, we shall cut the solution into two parts: a spatially localized part and the part near spatial infinity. For the localized part, we can use the local energy decay in Lemma 2.6, while for the part near infinity, we can view the metric as small perturbation of the Minkowski metric and use Lemma 2.2

To this end, we introduce a cutoff function $\phi \in C_{c}^{\infty}$ which equals 1 in the unit ball $B_{1}$ and support in $B_{2}$ and let $\phi_{R}(x)=\phi(x / R)$. Let $v=\phi_{R} u$ and $w=\left(1-\phi_{R}\right) u$ with $R \gg 1$ to be determined later, then

$$
\begin{gathered}
\square_{\mathfrak{g}} v=\left[-\Delta_{\mathfrak{g}}, \phi_{R}\right] u+\phi_{R} F:=f+\phi_{R} F . \\
\square_{\mathfrak{g}} w=-\left[-\Delta_{\mathfrak{g}}, \phi_{R}\right] u+\left(1-\phi_{R}\right) F:=-f+\left(1-\phi_{R}\right) F .
\end{gathered}
$$


By (2.12), we have

$$
\|f\|_{L_{t, x}^{2}} \lesssim\left\|\phi_{2 R} \partial_{x} u\right\|_{L_{t, x}^{2}}+\left\|\phi_{2 R} u\right\|_{L_{t, x}^{2}} \lesssim\left\|u_{0}\right\|_{\dot{H}_{x}^{1}}+\left\|u_{1}\right\|_{L_{x}^{2}}+\left\|\langle x\rangle^{1 / 2+\epsilon} F\right\|_{L_{T, x}^{2}} .
$$

Note that $v$ is compactly supported in $x$, so Lemma 2.6 applies, i.e.

$$
\|v\|_{L_{t}^{2} H_{x}^{1}}+\left\|\partial_{t} v\right\|_{L_{t, x}^{2}} \lesssim\left\|u_{0}\right\|_{\dot{H}_{x}^{1}}+\left\|u_{1}\right\|_{L_{x}^{2}}+\left\|\langle x\rangle^{1 / 2+\epsilon} F\right\|_{L_{T, x}^{2}} .
$$

If we define

$$
\tilde{g}^{i j}=\left(1-\phi_{R / 2}\right) g^{i j}+\phi_{R / 2} \delta^{i j}
$$

then

$$
\tilde{\Delta}=\sum_{i j} \tilde{g}^{-2} \partial_{i} \tilde{g}^{i j} \tilde{g}^{2} \partial_{j}=\Delta+h^{i j} \partial_{i} \partial_{j}+b^{i} \partial_{i}+c
$$

where $h^{i j}=\left(1-\phi_{R / 2}\right)\left(g^{i j}-\delta^{i j}\right), b^{i}=\left(1-\phi_{R / 4}\right) r_{1}^{i}$ and $c=\left(1-\phi_{R / 4}\right) r_{2}$, with the $r_{j}$ satisfying the bounds in (2.7). Thus since, $\tilde{\Delta} w=\Delta_{\mathfrak{g}} w, w$ satisfies

(2.18) $\left(\partial_{t}^{2}-\Delta-h^{i j}\right) w=\square_{\mathfrak{g}} w+\left(b^{i} \partial_{i}+c\right) w=\left(1-\phi_{R}\right) F-f+\left(b^{i} \partial_{i}+c\right) w:=G$.

Note that by (H1) and choosing $R \gg 1$ large enough, $h^{i j}$ will be small enough so that we can apply Lemma 2.2 with $\epsilon<\rho / 4$,

RHS of (2.3) $\lesssim\left\|w^{\prime}(0, \cdot)\right\|_{L^{2}\left(\mathbb{R}^{d}\right)}^{2}+\int_{0}^{T} \int\left(\left|w^{\prime}\right|+\frac{w}{|x|}\right)\left(|G|+\left(\left|h^{\prime}\right|+\frac{h}{|x|}\right)\left|w^{\prime}\right|\right) d x d t$

$$
\begin{aligned}
\lesssim & \left\|w^{\prime}(0, \cdot)\right\|_{L^{2}\left(\mathbb{R}^{d}\right)}^{2}+\left\|\langle x\rangle^{-1 / 2-\epsilon}\left(\left|w^{\prime}\right|+\frac{w}{|x|}\right)\right\|_{L_{T, x}^{2}}\left\|\langle x\rangle^{1 / 2+\epsilon} G\right\|_{L_{T, x}^{2}} \\
& +\left\|\langle x\rangle^{-1 / 2-\rho / 2}\left(\left|w^{\prime}\right|+\frac{w}{|x|}\right)\right\|_{L_{T, x}^{2}}^{2} \\
\lesssim & \left\|w^{\prime}(0, \cdot)\right\|_{L^{2}\left(\mathbb{R}^{d}\right)}^{2}+\varepsilon\left\|\langle x\rangle^{-1 / 2-\epsilon}\left(\left|w^{\prime}\right|+\frac{w}{|x|}\right)\right\|_{L_{T, x}^{2}}^{2}+\frac{1}{\varepsilon}\left\|\langle x\rangle^{1 / 2+\epsilon} G\right\|_{L_{T, x}^{2}}^{2} \\
& +R^{-\rho / 2}\left\|\langle x\rangle^{-1 / 2-\rho / 4}\left(\left|w^{\prime}\right|+\frac{w}{|x|}\right)\right\|_{L_{T, x}^{2}}^{2} \\
\lesssim & \left\|w^{\prime}(0, \cdot)\right\|_{L^{2}\left(\mathbb{R}^{d}\right)}^{2}+2 \varepsilon\left\|\langle x\rangle^{-1 / 2-\epsilon}\left(\left|w^{\prime}\right|+\frac{w}{|x|}\right)\right\|_{L_{T, x}^{2}}^{2}+\frac{1}{\varepsilon}\left\|\langle x\rangle^{1 / 2+\epsilon} G\right\|_{L_{T, x}^{2}}^{2} .
\end{aligned}
$$

Since we know from Lemma 2.2 ,

$\left\|\langle x\rangle^{-1 / 2-\epsilon}\left(\left|w^{\prime}\right|+\frac{|w|}{|x|}\right)\right\|_{L_{T, x}^{2}} \lesssim\left\|\langle x\rangle^{-1 / 2-\epsilon}\left|u^{\prime}\right|+\langle x\rangle^{-3 / 2-\epsilon}|u|\right\|_{L_{T, x}^{2}} \lesssim\left\|u^{\prime}(0)\right\|_{L_{x}^{2}}+\left\|\langle x\rangle^{1 / 2+\epsilon} F\right\|_{L_{T, x}^{2}}$,

and

$\left\|\langle x\rangle^{1 / 2+\epsilon}\left(b^{i} \partial_{i}+c\right) w\right\|_{L_{T, x}^{2}} \lesssim\left\|\langle x\rangle^{-1 / 2+\epsilon-\rho}\left(\left|w^{\prime}\right|+\frac{w}{|x|}\right)\right\|_{L_{T, x}^{2}} \lesssim\left\|\langle x\rangle^{-1 / 2-\epsilon}\left(\left|w^{\prime}\right|+\frac{w}{|x|}\right)\right\|_{L_{T, x}^{2}}$,

Thus combining (2.15),

$$
\text { RHS of (2.3) } \lesssim\left\|u_{0}\right\|_{\dot{H}^{1}}+\left\|u_{1}\right\|_{L_{x}^{2}}+\left\|\langle x\rangle^{1 / 2+\epsilon} F\right\|_{L_{T, x}^{2}} .
$$

Applying Lemma 2.2 and (2.16), we get finally that

$$
(\ln (2+T))^{-1 / 2}\left\|\langle x\rangle^{-1 / 2}\left(\left|u^{\prime}\right|+\frac{|u|}{\langle x\rangle}\right)\right\|_{L_{T, x}^{2}} \lesssim\left\|u_{0}\right\|_{\dot{H}^{1}}+\left\|u_{1}\right\|_{L_{x}^{2}}+\left\|\langle x\rangle^{1 / 2+\epsilon} F\right\|_{L_{T, x}^{2}},
$$

Therefore, we can use Lemma 2.2 to see that $w$ also satisfies the bounds in (1.6), which completes the proof of Theorem 1.2 


\section{Higher Order KSS Estimates}

In this section, we prove the KSS estimates involving high order derivatives as stated in Theorem 1.3.

We first give the KSS estimate for $P$. Consider the equation

$$
\left\{\begin{array}{l}
\left(\partial_{t}^{2}+P\right) v(t, x)=G(t, x), \quad(t, x) \in \mathbb{R}_{+} \times \mathbb{R}^{d} \\
u(0, x)=v_{0}(x), \quad \partial_{t} v(0, x)=v_{1}(x), \quad x \in \mathbb{R}^{d} .
\end{array}\right.
$$

Recall that if we let $v=g u$ and $G=g F$, then

$$
\left(\partial_{t}^{2}-\Delta_{\mathfrak{g}}\right) u=F \Leftrightarrow\left(\partial_{t}^{2}+P\right) v=G .
$$

Thus we have also the following KSS estimate

$$
A_{\mu}(T)\left(\left\|\langle x\rangle^{-\mu} v^{\prime}\right\|_{L_{T, x}^{2}}+\left\|\langle x\rangle^{-\mu-1} v\right\|_{L_{T, x}^{2}}\right) \lesssim\left\|v^{\prime}(0, \cdot)\right\|_{L_{x}^{2}}+\left\|\langle x\rangle^{\frac{1}{2}+\varepsilon} G\right\|_{L_{T, x}^{2}}
$$

for $\mu \geq 1 / 2$ and $\epsilon>0$.

For $\partial_{x}^{\alpha} \Omega^{\beta} v$, we will use induction on $|\beta|$ to give the proof. First, for $|\beta|=0$, we have

$$
\left(\partial_{t}^{2}+P\right) \partial_{x}^{\alpha} v=\partial_{x}^{\alpha} G+\left[P, \partial_{x}^{\alpha}\right] v .
$$

Locally, by ellipticity, we have

$$
\sum_{|\alpha|=2}\left\|\partial_{x}^{\alpha} f\right\|_{L_{|x| \leq R}^{2}} \lesssim \sum_{|\beta| \leq 1}\left\|\partial_{x}^{\beta} f\right\|_{L_{|x| \leq 2 R}^{2}}+\|P f\|_{L_{|x| \leq 2 R}^{2}},
$$

and so, by an induction argument, and (2.4) and (2.5) in Lemma 2.3.

$$
\begin{aligned}
\left\|\partial_{x}^{\alpha} f\right\|_{L_{|x| \leq R}^{2}} & \lesssim \sum_{2 j+1 \leq|\alpha|}\left\|\partial_{x} P^{j} f\right\|_{L_{|x| \leq 2 R}^{2}}+\sum_{2 j \leq|\alpha|}\left\|P^{j} f\right\|_{L_{|x| \leq 2 R}^{2}} \\
& \lesssim\left\|\langle x\rangle^{-2} f\right\|_{L^{2}}+\sum_{j \leq|\alpha|-1}\left\|\langle x\rangle^{-1} \partial_{x} P^{j / 2} f\right\|_{L^{2} .}
\end{aligned}
$$

Thus by the KSS estimates (3.3), (2.5) and (2.10),

$$
\begin{aligned}
\sum_{|\alpha| \leq N+1}\left\|\partial_{x}^{\alpha} v\right\|_{L_{t,|x| \leq R}^{2}} & \lesssim \sum_{j \leq N}\left\|\langle x\rangle^{-1} \partial_{x} P^{j / 2} v\right\|_{L_{t, x}^{2}}+\left\|\langle x\rangle^{-2} v\right\|_{L^{2}} \\
& \lesssim \sum_{j \leq N}\left(\left\|\partial_{x} P^{j / 2} v_{0}\right\|_{L^{2}}+\left\|P^{j / 2} v_{1}\right\|_{L^{2}}+\left\|\langle x\rangle^{1 / 2+\epsilon / 2} P^{j / 2} G\right\|_{L_{t, x}^{2}}\right) \\
(3.4) & \lesssim \sum_{\beta \leq N}\left(\left\|\partial_{x}^{\beta} v_{0}\right\|_{\dot{H}^{1}}+\left\|\partial_{x}^{\beta} v_{1}\right\|_{L^{2}}+\left\|\langle x\rangle^{1 / 2+\epsilon} \partial_{x}^{\beta} G\right\|_{L_{t, x}^{2}}\right) .
\end{aligned}
$$

Using again (3.3), we have

$$
A_{\mu}(T) \sum_{|\alpha| \leq N}\left\|\langle x\rangle^{-\mu}\left(\left|\left(\partial_{x}^{\alpha} v\right)^{\prime}\right|+\frac{\left|\partial_{x}^{\alpha} v\right|}{\langle x\rangle}\right)\right\|_{L_{T, x}^{2}} \lesssim
$$

$$
\sum_{|\alpha| \leq N}\left(\left\|\partial_{x}^{\alpha} v_{0}\right\|_{\dot{H}^{1}}+\left\|\partial_{x}^{\alpha} v_{1}\right\|_{L_{x}^{2}}+\left\|\langle x\rangle^{1 / 2+\epsilon} \partial_{x}^{\alpha} G\right\|_{L_{T, x}^{2}}+\left\|\langle x\rangle^{1 / 2+\epsilon}\left[P, \partial_{x}^{\alpha}\right] v\right\|_{L_{T, x}^{2}}\right)
$$

for $\mu \geq 1 / 2$ and $\epsilon>0$. Note that

$$
\left|\left[P, \partial_{x}^{\alpha}\right] v\right| \lesssim\langle x\rangle^{-\rho-1} \sum_{1 \leq|\gamma| \leq|\alpha|+1}\left|\partial_{x}^{\gamma} v\right|+\langle x\rangle^{-\rho-2}|v|,
$$


if choose $\epsilon>0$ small enough so that $1 / 2+\epsilon-\rho-1<-1 / 2-3 \epsilon$ and $R \gg 1$,

$$
\sum_{|\alpha| \leq N}\left\|\langle x\rangle^{1 / 2+\epsilon}\left[P, \partial_{x}^{\alpha}\right] v\right\|_{L_{T, x}^{2}} \lesssim \varepsilon \sum_{|\alpha| \leq N}\left\|\langle x\rangle^{-1 / 2-\epsilon}\left(\left|\left(\partial_{x}^{\alpha} v\right)^{\prime}\right|+\frac{\left|\partial_{x}^{\alpha} v\right|}{\langle x\rangle}\right)\right\|+\sum_{|\alpha| \leq N+1}\left\|\partial_{x}^{\alpha} v\right\|_{L_{t}^{2} L_{|x| \leq R}^{2}} .
$$

So the first term in the right hand side of the inequality can be absorbed by (3.5) with $\mu=1 / 2+\epsilon$. Thus for $\rho>0, \mu \geq 1 / 2$ and $\epsilon>0$,

$A_{\mu}(T) \sum_{|\alpha| \leq N}\left\|\langle x\rangle^{-\mu}\left(\left|\left(\partial_{x}^{\alpha} v\right)^{\prime}\right|+\frac{\left|\partial_{x}^{\alpha} v\right|}{\langle x\rangle}\right)\right\|_{L_{T, x}^{2}} \lesssim \sum_{|\alpha| \leq N}\left(\left\|\partial_{x}^{\alpha} v_{0}\right\|_{\dot{H}^{1}}+\left\|\partial_{x}^{\alpha} v_{1}\right\|_{L_{x}^{2}}+\left\|\langle x\rangle^{1 / 2+\epsilon} \partial_{x}^{\alpha} G\right\|_{L_{T, x}^{2}}\right)$.

Now we claim that for $\rho>1, \mu \geq 1 / 2$ and $\epsilon>0$, we have

$$
\begin{array}{r}
A_{\mu}(T) \sum_{|\alpha|+|\beta| \leq N}\left\|\langle x\rangle^{-\mu}\left(\left|\left(\partial_{x}^{\alpha} \Omega^{\beta} v\right)^{\prime}\right|+\frac{\left|\partial_{x}^{\alpha} \Omega^{\beta} v\right|}{\langle x\rangle}\right)\right\|_{L_{T, x}^{2}} \lesssim \\
\sum_{|\alpha|+|\beta| \leq N}\left(\left\|\partial_{x}^{\alpha} \Omega^{\beta} v_{0}\right\|_{\dot{H}^{1}}+\left\|\partial_{x}^{\alpha} \Omega^{\beta} v_{1}\right\|_{L_{x}^{2}}+\left\|\langle x\rangle^{1 / 2+\epsilon} \partial_{x}^{\alpha} \Omega^{\beta} G\right\|_{L_{T, x}^{2}}\right) .
\end{array}
$$

We use induction on $|\beta|$ to give the proof. Assume the estimate (3.7) is true for $|\beta| \leq k$, then

$$
\left(\partial_{t}^{2}+P\right) \Omega v=\Omega G+[P, \Omega] v
$$

and we are reduced to estimate

$$
\sum_{|\alpha|+|\beta| \leq N-1,|\beta| \leq k}\left\|\langle x\rangle^{1 / 2+\epsilon} \partial_{x}^{\alpha} \Omega^{\beta}[P, \Omega] v\right\|_{L_{T, x}^{2}}
$$

by (3.7). Note that

$$
[P, \Omega] v=\sum_{|\gamma| \leq 2} r_{|\gamma|-2} \partial_{x}^{\gamma} v,
$$

where $r_{j}$ are functions satisfying (2.7). Thus if $\rho>1$ and $\epsilon>0$ small enough such that $\rho>1+2 \epsilon$,

$$
\begin{aligned}
& \sum_{|\alpha|+|\beta| \leq N-1,|\beta| \leq k}\left\|\langle x\rangle^{1 / 2+\epsilon} \partial_{x}^{\alpha} \Omega^{\beta}[P, \Omega] v\right\|_{L_{T, x}^{2}} \\
& \lesssim \sum_{|\alpha|+|\beta| \leq N,|\beta| \leq k}\left\|\langle x\rangle^{1 / 2+\epsilon-\rho}\left(\left|\left(\partial_{x}^{\alpha} \Omega^{\beta} v\right)^{\prime}\right|+\frac{|v|}{\langle x\rangle}\right)\right\|_{L_{T, x}^{2}} \\
& \lesssim \sum_{|\alpha|+|\beta| \leq N,|\beta| \leq k}\left\|\langle x\rangle^{-1 / 2-\epsilon}\left(\left|\left(\partial_{x}^{\alpha} \Omega^{\beta} v\right)^{\prime}\right|+\frac{|v|}{\langle x\rangle}\right)\right\|_{L_{T, x}^{2}} \lesssim(\underline{3.7}) .
\end{aligned}
$$

Since $\partial_{t}$ commutate with $\partial_{t}^{2}+P$, we can conclude that for $\rho>1, \mu \geq 1 / 2$ and $\epsilon>0$, we have

$$
\begin{gathered}
A_{\mu}(T) \sum_{j+|\alpha|+|\beta| \leq N}\left\|\langle x\rangle^{-\mu}\left(\left|\left(\partial_{t}^{j} \partial_{x}^{\alpha} \Omega^{\beta} v\right)^{\prime}\right|+\frac{\left|\partial_{t}^{j} \partial_{x}^{\alpha} \Omega^{\beta} v\right|}{\langle x\rangle}\right)\right\|_{L_{T, x}^{2}} \lesssim \\
\sum_{j+|\alpha|+|\beta| \leq N}\left(\left\|\left(\partial_{t}^{j} \partial_{x}^{\alpha} \Omega^{\beta} v\right)^{\prime}(0, \cdot)\right\|_{L^{2}}+\left\|\langle x\rangle^{1 / 2+\epsilon} \partial_{t}^{j} \partial_{x}^{\alpha} \Omega^{\beta} G\right\|_{L_{T, x}^{2}}\right) .
\end{gathered}
$$


Combining the energy estimates, we get the following estimates

$$
\begin{aligned}
& \sup _{0 \leq t \leq T} \sum_{1 \leq k+j \leq N+1}\left\|\partial_{t}^{k} P^{j / 2} v(t, \cdot)\right\|_{L_{x}^{2}}+\sum_{|\alpha| \leq N} A_{\mu}(T)\left\|\langle x\rangle^{-\mu}\left(\left|\left(Z^{\alpha} v\right)^{\prime}\right|+\frac{\left|Z^{\alpha} v\right|}{\langle x\rangle}\right)\right\|_{L_{T}^{2} L_{x}^{2}} \\
& \lesssim \sum_{|\alpha| \leq N}\left(\left\|\left(Z^{\alpha} v\right)^{\prime}(0, \cdot)\right\|_{L_{x}^{2}}+\int_{0}^{T}\left\|Z^{\alpha} G(s, \cdot)\right\|_{L_{x}^{2}} d s\right) .
\end{aligned}
$$

for the solution to the equation (3.1). For the $\left\|\left(Z^{\alpha} v\right)^{\prime}(0, \cdot)\right\|_{L_{x}^{2}}$ part, if it has $\partial_{t}^{j}$ component with $j \geq 2$, we can use the equation to reduce it to the case $\partial_{t}^{j-2}$, with an additional term

$$
\sum_{|\alpha| \leq N-1}\left\|Z^{\alpha} G(0, \cdot)\right\|_{L_{x}^{2}} \lesssim \sum_{|\alpha| \leq N-1}\left\|Z^{\alpha} G(t, \cdot)\right\|_{L_{x}^{2} W_{t}^{1,1}} \lesssim \sum_{|\alpha| \leq N}\left\|Z^{\alpha} G(t, \cdot)\right\|_{L_{T}^{1} L_{x}^{2}} .
$$

This means that

$$
\begin{aligned}
\sup _{0 \leq t \leq T} \sum_{1 \leq k+j \leq N+1}\left\|\partial_{t}^{k} P^{j / 2} v(t, \cdot)\right\|_{L_{x}^{2}}+\sum_{|\alpha| \leq N} A_{\mu}(T)\left\|\langle x\rangle^{-\mu}\left(\left|\left(Z^{\alpha} v\right)^{\prime}\right|+\frac{\left|Z^{\alpha} v\right|}{\langle x\rangle}\right)\right\|_{L_{T}^{2} L_{x}^{2}} \\
\\
\qquad \sum_{|\alpha| \leq N}\left\|\left(Y^{\alpha} v\right)^{\prime}(0, \cdot)\right\|_{L_{x}^{2}}+\sum_{|\alpha| \leq N} \int_{0}^{T}\left\|Z^{\alpha} G(s, \cdot)\right\|_{L_{x}^{2}} d s .
\end{aligned}
$$

Turning back to the equation (1.5), let $v=g u$ and $G=g F$, then $\left(\partial_{t}^{2}+P^{2}\right) v=G$ with $v_{0}=g u_{0}, v_{1}=g u_{1}$. Note that

$$
\partial_{x} u=g^{-1} \partial_{x} g u-g^{-1}\left(\partial_{x} g\right) u=g^{-1} \partial_{x} v-g^{-2}\left(\partial_{x} g\right) v,
$$

we have

$$
\sum_{|\alpha| \leq N}\left\|\langle x\rangle^{-\mu}\left(\left|\left(Z^{\alpha} u\right)^{\prime}\right|+\frac{\left|Z^{\alpha} u\right|}{\langle x\rangle}\right)\right\|_{L_{T, x}^{2}} \lesssim \sum_{|\alpha| \leq N}\left\|\langle x\rangle^{-\mu}\left(\left|\left(Z^{\alpha} v\right)^{\prime}\right|+\frac{\left|Z^{\alpha} v\right|}{\langle x\rangle}\right)\right\|_{L_{T, x}^{2}} .
$$

This concludes the proof of Theorem 1.3 .

\section{Almost Global Existence}

In this section we shall prove one of our main existence theorems, Theorem 1.1 The proof will be similar to that of Keel, Smith and Sogge for the Minkowski case (see [14]). We start with the now standard Sobolev estimate (see [18]).

Lemma 4.1. Suppose that $h \in C^{\infty}\left(\mathbb{R}^{3}\right)$. Then, for $R>1$,

$$
\|h\|_{L^{\infty}(R / 2 \leq|x| \leq R)} \lesssim R^{-1} \sum_{|\alpha| \leq 2}\left\|Y^{\alpha} h\right\|_{L^{2}(R / 4 \leq|x| \leq 2 R)} .
$$

We now define the bilinear form $\widetilde{Q}$ by $\widetilde{Q}\left(u^{\prime}, u^{\prime}\right)=Q\left(u^{\prime}\right)$. The following estimate for the nonlinear part will be crucial.

Lemma 4.2. We have

$$
\sum_{|\alpha| \leq 4}\left\|Z^{\alpha} \widetilde{Q}\left(u^{\prime}, v^{\prime}\right)\right\|_{L^{2}\left(\mathbb{R}^{3}\right)}^{2} \lesssim\left(\sum_{|\alpha| \leq 4}\left\|\langle x\rangle^{-1 / 2} Z^{\alpha} u^{\prime}\right\|_{L^{2}\left(\mathbb{R}^{3}\right)}^{2}\right)\left(\sum_{|\alpha| \leq 4}\left\|\langle x\rangle^{-1 / 2} Z^{\alpha} v^{\prime}\right\|_{L^{2}\left(\mathbb{R}^{3}\right)}^{2}\right) .
$$


Proof. We clearly have the pointwise bound:

$$
\begin{aligned}
\left|Z^{\beta} \widetilde{Q}\left(u^{\prime}, v^{\prime}\right)(s, x)\right| \lesssim & \left(\sum_{|\alpha| \leq 4}\left|Z^{\alpha} u^{\prime}(s, x)\right|\right)\left(\sum_{|\alpha| \leq 2}\left|Z^{\alpha} v^{\prime}(s, x)\right|\right) \\
& +\left(\sum_{|\alpha| \leq 4}\left|Z^{\alpha} v^{\prime}(s, x)\right|\right)\left(\sum_{|\alpha| \leq 2}\left|Z^{\alpha} u^{\prime}(s, x)\right|\right) .
\end{aligned}
$$

We need only to estimate the first term. Using Lemma 4.1 for a given $R=2^{j}$, $j \geq 0$, we get

$$
\begin{aligned}
\left\|Z^{\beta} \widetilde{Q}\left(u^{\prime}, v^{\prime}\right)\right\|_{L^{2}\left(\left\{|x| \in\left[2^{j}, 2^{j+1}\right]\right\}\right)}^{2} & \\
& \lesssim 2^{-2 j} \sum_{|\alpha| \leq 4}\left\|Z^{\alpha} u^{\prime}\right\|_{L^{2}\left(\left\{|x| \in\left[2^{j}, 2^{j+1}\right]\right\}\right)}^{2} \sum_{|\alpha| \leq 4}\left\|Z^{\alpha} v^{\prime}\right\|_{L^{2}\left(\left\{|x| \in\left[2^{j-1}, 2^{j+2}\right]\right\}\right)}^{2} \\
& \lesssim \sum_{|\alpha| \leq 4}\left\|\langle x\rangle^{-1 / 2} Z^{\alpha} u^{\prime}\right\|_{L^{2}\left(\left\{|x| \in\left[2^{j}, 2^{j+1}\right]\right\}\right)}^{2} \sum_{|\alpha| \leq 4}\left\|\langle x\rangle^{-1 / 2} Z^{\alpha} v^{\prime}\right\|_{L^{2}\left(\left\{|x| \in\left[2^{j-1}, 2^{j+2}\right]\right\}\right)}^{2} \\
& \lesssim \sum_{|\alpha| \leq 4}\left\|\langle x\rangle^{-1 / 2} Z^{\alpha} u^{\prime}\right\|_{L^{2}\left(\left\{|x| \in\left[2^{j}, 2^{j+1}\right]\right\}\right)}^{2} \sum_{|\alpha| \leq 4}\left\|\langle x\rangle^{-1 / 2} Z^{\alpha} v^{\prime}\right\|_{L^{2}\left(\mathbb{R}^{3}\right)^{2}}^{2}
\end{aligned}
$$

We also have the bound

$$
\left\|Z^{\beta} \widetilde{Q}\left(u^{\prime}, v^{\prime}\right)\right\|_{L^{2}(\{|x|<1\})}^{2} \lesssim \sum_{|\alpha| \leq L}\left\|Z^{\alpha} u^{\prime}\right\|_{L^{2}(\{|x|<2\})}^{2} \sum_{|\alpha| \leq L}\left\|Z^{\alpha} v^{\prime}\right\|_{L^{2}(\{|x|<2\})}^{2} .
$$

Summing over $j$ gives the lemma.

Proof of Theorem 1.1. We follow [14. Let $u_{-1}=0$. We define $u_{k}, k \in \mathbb{N}$ inductively by letting $u_{k}$ to solve

$$
\left\{\begin{array}{l}
\square_{\mathfrak{g}} u_{k}=Q\left(u_{k-1}^{\prime}\right), \\
u(0, x)=u_{0}(x), \quad \partial_{t} u(0, x)=u_{1}(x) .
\end{array}\right.
$$

For $T>0$, we denote

$$
M_{k}(T)=\sup _{0 \leq t \leq T} \sum_{1 \leq i+j \leq 5}\left\|\partial_{t}^{i} P^{j / 2} g u_{k}\right\|_{L^{2}\left(\mathbb{R}^{3}\right)}+\sum_{|\alpha| \leq 4}(\ln (2+T))^{-1 / 2}\left\|\langle x\rangle^{-1 / 2} Z^{\alpha} u_{k}^{\prime}\right\|_{L^{2}\left([0, T] \times \mathbb{R}^{3}\right)} .
$$

Using Theorem 1.3. we see that there exists a constant $C_{0}$ such that

$$
M_{0}(T) \leq C_{0} \delta
$$

for any $T$. We claim that, for $k \geq 1$, we have

$$
M_{k}\left(T_{\delta}\right) \leq 2 C_{0} \delta
$$

for $\delta$ sufficiently small and $T_{\delta}$ appropriately chosen later. We will prove this inductively. Assume that the bound holds for $k-1$. By Theorem 1.3. we have, for $\delta$ 
small enough,

$$
\begin{aligned}
M_{k}\left(T_{\delta}\right) & \leq C_{0} \delta+C \sum_{|\alpha| \leq 4} \int_{0}^{T_{\delta}}\left\|Z^{\alpha} Q\left(u_{k-1}^{\prime}\right)(s, \cdot)\right\|_{L^{2}\left(\mathbb{R}^{d}\right)} d s \\
& \leq C_{0} \delta+C \sum_{|\alpha| \leq 4} \int_{0}^{T_{\delta}}\left\|\langle x\rangle^{-1 / 2} Z^{\alpha} u_{k-1}^{\prime}\right\|_{L^{2}\left(\mathbb{R}^{d}\right)}^{2} d s \\
& \leq C_{0} \delta+C \ln \left(2+T_{\delta}\right) M_{k-1}^{2}\left(T_{\delta}\right) \\
& \leq C_{0} \delta+C \ln \left(2+T_{\delta}\right)\left(2 C_{0} \delta\right)^{2},
\end{aligned}
$$

where we have also used Lemma 4.2 and the induction hypothesis. Then, to prove (4.3), it is enough to have

$$
C_{0} \delta+C \ln \left(2+T_{\delta}\right)\left(2 C_{0} \delta\right)^{2} \leq 2 C_{0} \delta \Longleftrightarrow 4 C C_{0} \ln \left(2+T_{\delta}\right) \delta \leq 1
$$

Therefore, we can set $T_{\delta}=\exp (c / \delta)$ and $c$ small enough.

To show that the sequence $u_{k}$ converges, we estimate the quantity

$$
\begin{aligned}
A_{k}(T)= & \sup _{0 \leq t \leq T} \sum_{1 \leq i+j \leq 5}\left\|\partial_{t}^{i} P^{j / 2} g\left(u_{k}-u_{k-1}\right)\right\|_{L^{2}\left(\mathbb{R}^{d}\right)} \\
& +\sum_{|\alpha| \leq 4}\left(\ln \left(2+T_{\delta}\right)\right)^{-1 / 2}\left\|\langle x\rangle^{-1 / 2} Z^{\alpha}\left(u_{k}^{\prime}-u_{k-1}^{\prime}\right)\right\|_{L^{2}\left([0, T] \times \mathbb{R}^{d}\right)} .
\end{aligned}
$$

It is clearly sufficient to show

$$
A_{k}(T) \leq \frac{1}{2} A_{k-1}(T)
$$

Using Lemma 4.2 and repeating the above arguments, we obtain

$$
\begin{aligned}
A_{k}\left(T_{\delta}\right) & \leq \widetilde{C} \sum_{|\alpha| \leq 4} \int_{0}^{T_{\delta}}\left\|\langle x\rangle^{-1 / 2} Z^{\alpha}\left(u_{k-1}^{\prime}-u_{k-2}^{\prime}\right)\right\|_{L^{2}\left(\mathbb{R}^{d}\right)} \\
& \times \sum_{|\alpha| \leq 4}\left(\left\|\langle x\rangle^{-1 / 2} Z^{\alpha} u_{k-1}^{\prime}\right\|_{L^{2}\left(\mathbb{R}^{d}\right)}+\left\|\langle x\rangle^{-1 / 2} Z^{\alpha} u_{k-2}^{\prime}\right\|_{L^{2}\left(\mathbb{R}^{d}\right)}\right) d s \\
& \leq \widetilde{C} \ln \left(2+T_{\delta}\right)\left(M_{k-1}\left(T_{\delta}\right)+M_{k-2}\left(T_{\delta}\right)\right) A_{k-1}\left(T_{\delta}\right) .
\end{aligned}
$$

Using (4.3), the above inequality leads to (4.5) if $\delta$ is small enough.

\section{Global Strichartz Estimates}

In this section we shall prove Theorem 1.4, the global Strichartz estimates for $\square_{\mathfrak{g}}$. The proof will follow closely the arguments of [30], 4] and [9, which dealt with compact perturbations of the Minkowski metric or obstacle case.

Recall that we have equivalence (3.2) between the wave equations involving $P$ and $-\Delta_{\mathfrak{g}}$. We need only to give the proof for the case of following equation,

$$
\left\{\begin{array}{l}
\left(\partial_{t}^{2}+P\right) u(t, x)=F(t, x), \quad(t, x) \in \mathbb{R}_{+} \times \mathbb{R}^{d} \\
u(0, x)=u_{0}(x), \quad \partial_{t} u(0, x)=u_{1}(x), \quad x \in \mathbb{R}^{d} .
\end{array}\right.
$$

As in [9], we will need the following four ingredients: local energy decay for $\partial_{t}^{2}+P$, local Strichartz estimates for $\partial_{t}^{2}+P$, global Strichartz estimates for $\partial_{t}^{2}+\tilde{P}$ with $\tilde{\mathfrak{g}}=\mathfrak{g}$ in $|x| \geq R$ for some $R>0$, and an estimate for $\partial_{t}^{2}+\tilde{P}$ like Proposition 2.1 in 9 . 
Let us give first the estimates for $\partial_{t}^{2}+\tilde{P}$, where $\tilde{\mathfrak{g}}$ is defined by (2.17), i.e., $\tilde{g}^{i j}=\left(1-\phi_{R / 2}\right) g^{i j}+\phi_{R / 2} \delta^{i j}$. Then

$$
\tilde{P}=-\sum_{i j} \frac{1}{\tilde{g}} \partial_{i} \tilde{g}^{i j} \tilde{g}^{2} \partial_{j} \frac{1}{\tilde{g}}=-\partial_{i} \tilde{g}^{i j} \partial_{j}+b^{i} \partial_{i}+c
$$

where $b^{i}=-2 \tilde{g}^{-1} \partial_{j}\left(\tilde{g} \tilde{g}^{i j}\right)-\tilde{g} \tilde{g}^{i j} \partial_{j} \tilde{g}^{-1}+\partial_{j} \tilde{g}^{i j}$ and $c=\tilde{g}^{-1} \partial_{i}\left(\tilde{g}^{i j} \partial_{j} \tilde{g}\right)$.

Consider the wave equation

$$
\left\{\begin{array}{l}
\left(\partial_{t}^{2}+\tilde{P}\right) u=0, \quad(t, x) \in \mathbb{R}_{+} \times \mathbb{R}^{d} \\
u(0, x)=u_{0}(x), \quad \partial_{t} u(0, x)=u_{1}(x), \quad x \in \mathbb{R}^{d} .
\end{array}\right.
$$

We denote

$$
A_{j}=\mathbb{R} \times\left\{2^{j} \leq|x| \leq 2^{j+1}\right\}, \quad A_{<j}=\mathbb{R} \times\left\{|x| \leq 2^{j}\right\} .
$$

By hypotheses ( $\mathrm{H1}$ ), we can see that if $R \gg 1$ so that $R^{-\rho} \leq \epsilon$,

$$
\sum_{j \in \mathbb{Z}} \sup _{A_{j}}|x|^{2}\left|\nabla^{2} \tilde{g}^{i j}(x)\right|+|x|\left|\nabla \tilde{g}^{i j}(x)\right|+\left|\tilde{g}^{i j}(x)-\delta^{i j}\right| \lesssim \sum_{j \gtrsim \ln (R)} \sup _{A_{j}}|x|^{-\rho} \lesssim R^{-\rho} \leq \epsilon
$$

and, for the lower order terms,

$$
\begin{gathered}
\sum_{j \in \mathbb{Z}} \sup _{A_{j}}|x|^{2}\left|\nabla b^{i}(x)\right|+|x|\left|b^{i}(x)\right| \lesssim \sum_{j \gtrsim \ln (R)} \sup _{A_{j}}|x|^{-\rho} \leq \epsilon, \\
\sum_{j \in \mathbb{Z}} \sup _{A_{j}}|x|^{4}|c(t, x)|^{2} \lesssim \sum_{j \gtrsim \ln (R)} \sup _{A_{j}}|x|^{-2 \rho} \leq \epsilon .
\end{gathered}
$$

Thus we can apply Theorem 6 in Metcalfe-Tataru 24] to get the following

Proposition 5.1 (Global Strichartz Estimates for Small Perturbation). Let $d \geq 3$, $s \in[0,1](s \in(0,1)$ if $d=3)$. The solution of (5.2) satisfies

$$
\|u\|_{L_{t}^{q} L_{x}^{r}} \lesssim\left\|u_{0}\right\|_{\dot{H}^{s}}+\left\|u_{1}\right\|_{\dot{H}^{s-1}},
$$

for any admissible $(s, q, r)$ with $q>2, r<\infty$. In other words, we have

$$
\left\|e^{i t \tilde{P}^{1 / 2}} f\right\|_{L_{t}^{q} L_{x}^{r}} \lesssim\|f\|_{\dot{H}^{s}},
$$

As a corollary, we can get an estimate for $\partial_{t}^{2}+\tilde{P}$ like Proposition 2.1 in [9].

Proposition 5.2. Let $d \geq 3, s \in[0,1](s \in(0,1)$ if $d=3)$. The solution $u$ of $\left(\partial_{t}^{2}+\tilde{P}\right) u=\beta F=F$ satisfies

$$
\|u\|_{L_{t}^{q} L_{x}^{r}} \lesssim\left\|u_{0}\right\|_{\dot{H}^{s}}+\left\|u_{1}\right\|_{\dot{H}^{s-1}}+\|F\|_{L_{t}^{2} H^{s-1}},
$$

for any admissible $(s, q, r)$ with $q>2, r<\infty$.

Proof. Applying (2.14) in this setting, we know that for any $s \in[0,1]$,

$$
\left\|\beta e^{i t \tilde{P}^{1 / 2}} f\right\|_{L_{t}^{2} H^{s}} \lesssim\|f\|_{\dot{H}^{s}} .
$$

By duality, this is equivalent to say that for $s \in[-1,0]$,

$$
\left\|\int e^{-i t \tilde{P}^{1 / 2}} \beta F(t, \cdot) d t\right\|_{\dot{H}^{s}} \lesssim\|f\|_{L_{t}^{2} H^{s}}
$$


Combining (5.7) and Lemma 2.4 (2.8),

$$
\begin{aligned}
\| \int e^{i(t-s) \tilde{P}^{1 / 2} \tilde{P}^{-1 / 2} \beta F(s, \cdot) d s \|_{L_{t}^{q} L_{x}^{r}}} & \lesssim\left\|\int e^{-i s \tilde{P}^{1 / 2}} \tilde{P}^{-1 / 2} \beta F(s, \cdot) d s\right\|_{\dot{H}^{s}} \\
& \lesssim\left\|\int e^{-i s \tilde{P}^{1 / 2}} \beta F(s, \cdot) d s\right\|_{\dot{H}^{s-1}} \\
& \lesssim\|F\|_{L_{t}^{2} H^{s-1}} .
\end{aligned}
$$

Since $q>2$, we can apply the Christ-Kiselev lemma [5] to conclude the proof.

Now we give the local Strichartz estimates for the $\partial_{t}^{2}+P$. Consider the wave equation

$$
\left\{\begin{array}{l}
\left(\partial_{t}^{2}+P\right) u(t, x)=0, \quad(t, x) \in \mathbb{R}_{+} \times \mathbb{R}^{d} \\
u(0, x)=u_{0}(x), \quad \partial_{t} u(0, x)=u_{1}(x), \quad x \in \mathbb{R}^{d} .
\end{array}\right.
$$

The local Strichartz estimates for the variable coefficient wave equations was studied extensively, see e.g. Kapitanski [13], Mockenhaupt-Seeger-Sogge 25], Smith [29], Bahouri-Chemin [1] 2, Tataru 34 [35] 37. In particular, we have

Lemma 5.3 (Theroem 1.1 in Tataru [37]). If $\left(\partial_{t}, \partial_{x}\right)^{\alpha} a^{i j}(t, x) \in L_{t \in[0,1]}^{1} L_{x}^{\infty}$ for any $|\alpha|=2$ and $\left(\partial_{t}^{2}-\partial_{i} a^{i j}(t, x) \partial_{j}\right) u=F$, then

$$
\left\|D^{1-s} u\right\|_{L_{t \in[0,1]}^{q} L_{x}^{r}} \lesssim\left\|u^{\prime}\right\|_{L_{t \in[0,1]}^{\infty} L_{x}^{2}}+\|F\|_{L_{t \in[0,1]}^{1} L_{x}^{2}}
$$

for any admissible $(s, q, r)$ with $r<\infty$.

Note that we can write

$$
\partial_{t}^{2}+P=\partial_{t}^{2}-\partial_{i} g^{i j} \partial_{j}+b^{i} \partial_{i}+c
$$

with $b^{i}=\mathcal{O}\left(\langle x\rangle^{-1-\rho}\right)$ and $c=\mathcal{O}\left(\langle x\rangle^{-2-\rho}\right)$. Moreover, for $a^{i j}=g^{i j}$ and $|\alpha|=2$, we have $\partial_{x}^{\alpha} a^{i j}(x)=\mathcal{O}\left(\langle x\rangle^{-2-\rho}\right) \in L_{t \in[0,1]}^{1} L_{x}^{\infty}$. Then for the equation (5.9), we have

$$
\begin{aligned}
\left\|D^{1-s} u\right\|_{L_{t \in[0,1]}^{q} L_{x}^{r}} & \lesssim\left\|u^{\prime}\right\|_{L_{t \in[0,1]}^{\infty} L_{x}^{2}}+\left\|b^{i} \partial_{i} u+c u\right\|_{L_{t}^{1} L_{x}^{2}} \\
& \lesssim\left\|u^{\prime}\right\|_{L_{t \in[0,1]}^{\infty} L_{x}^{2}} \\
& \lesssim\left\|u_{0}\right\|_{\dot{H}_{x}^{1}}+\left\|u_{1}\right\|_{L_{x}^{2}}
\end{aligned}
$$

by energy estimates and Lemma 5.3 . Moreover, we can prove the following

Proposition 5.4 (Local Strichartz Estimates). Let $s \in[0,1],(s, q, r)$ admissible with $r<\infty$, and $u$ be the solution to the equation (5.9), then

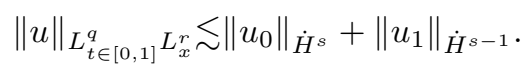

Proof. Since $\left(\partial_{t}^{2}+P\right) u=0$, we have $\left(\partial_{t}^{2}+P\right) P^{\frac{s-1}{2}} u=0$. Thus by (2.11) and (2.8) in Lemma 2.4 and (5.10),

$$
\begin{aligned}
\|u\|_{L_{t \in[0,1]}^{q} L_{x}^{r}} & \lesssim\left\|P^{\frac{1-s}{2}} P^{\frac{s-1}{2}} u\right\|_{L_{t \in[0,1]}^{q} L_{x}^{r}} \\
& \lesssim\left\|D^{1-s} P^{\frac{s-1}{2}} u\right\|_{L_{t \in[0,1]}^{q} L_{x}^{r}} \\
& \lesssim\left\|P^{\frac{s-1}{2}} u_{0}\right\|_{\dot{H}^{1}}+\left\|P^{\frac{s-1}{2}} u_{1}\right\|_{L^{2}} \\
& \lesssim\left\|u_{0}\right\|_{\dot{H}^{s}}+\left\|u_{1}\right\|_{\dot{H}^{s-1}},
\end{aligned}
$$


if $(s, q, r)$ is admissible, $s \in[0,1]$ and $r<\frac{d}{1-s}$, i.e., $s>1-\frac{d}{r}$. Since $(s, q, r)$ is admissible, then if $d=3$, we must have $q>2$,

$$
s=\frac{d}{2}-\frac{d}{r}-\frac{1}{q}>1-\frac{d}{r} .
$$

Similarly, if $d \geq 4$, we have $q \geq 2$,

$$
s=\frac{d}{2}-\frac{d}{r}-\frac{1}{q} \geq \frac{d-1}{2}-\frac{d}{r}>1-\frac{d}{r} .
$$

This means that we have the local Strichartz estimates for all the cases where $(s, q, r)$ is admissible and $s \in[0,1]$.

Now we are ready to give the proof of the global Strichartz estimates for $\partial_{t}^{2}+P$.

Proposition 5.5. For the linear equation (5.1), assume that

$$
u_{0}(x)=u_{1}(x)=F(t, x)=0 \quad \text { when }|x|>2 R,
$$

then for any $s \in[0,1]$ and admissible $(s, q, r)$ with $q>2$ and $r<\infty$, we have

$$
\|u\|_{L_{t}^{q} L_{x}^{r}} \lesssim\left\|u_{0}\right\|_{\dot{H}^{s}}+\left\|u_{1}\right\|_{\dot{H}_{x}^{s-1}}+\|F\|_{L_{t}^{2} \dot{H}_{x}^{s-1}} .
$$

Proof. One of the key ingredients in the proof is the following variant of Lemma 2.6. which holds for all $s \in[0,1]$,

$$
\|\beta u\|_{L_{t}^{2} \dot{H}^{s} \cap L_{t}^{\infty} \dot{H}^{s}}+\left\|\beta u_{t}\right\|_{L_{t}^{2} \dot{H}^{s-1} \cap L_{t}^{\infty} \dot{H}^{s-1}} \lesssim\left\|u_{0}\right\|_{\dot{H}^{s}}+\left\|u_{1}\right\|_{\dot{H}_{x}^{s-1}}+\|F\|_{L_{t}^{2} \dot{H}_{x}^{s-1}} .
$$

The $L_{t}^{2}$ estimate with $s=1$ comes from (2.13), then the estimates follow from Duhamel's formula and duality.

To prove (5.12), let us argue as before. Let $v=\phi_{3 R} u$ and $w=\left(1-\phi_{3 R}\right) u$ with $R \gg 1$ as in the definition of $\tilde{g}$. Then $w$ solves the wave equation for $\partial_{t}^{2}+\tilde{P}$,

$$
\left\{\begin{array}{l}
\left(\partial_{t}^{2}+\tilde{P}\right) w=\left(\partial_{t}^{2}+P\right) w=\left[\phi_{3 R}, P\right] u \\
\left.w\right|_{t=0}=\left.\partial_{t} w\right|_{t=0}=0
\end{array}\right.
$$

An application of Proposition 5.2 shows that $\|w\|_{L_{t}^{q} L_{x}^{r}}$ is dominated by $\|\beta u\|_{L_{t}^{2} \dot{H}^{s}}$ if $\beta$ equals one on the support of $\phi_{3 R}$. Therefore, by (5.13), $\|w\|_{L_{t}^{q} L_{x}^{r}}$ is dominated by the right side of (5.12).

As a result, we are left with showing that if $v=\phi_{3 R} u$ then

$$
\|v\|_{L_{t}^{q} L_{x}^{r}} \lesssim\left\|u_{0}\right\|_{\dot{H}^{s}}+\|g\|_{\dot{H}^{s-1}}+\|F\|_{L_{t}^{2} \dot{H}^{s-1}} .
$$

To do this, fix $\varphi \in C_{c}^{\infty}((-1,1))$ satisfying $\sum_{j=-\infty}^{\infty} \varphi(t-j)=1$. For a given $j \in \mathbb{N}$, let $v_{j}=\varphi(t-j) v$. Then $v_{j}$ solves

$$
\left\{\begin{array}{l}
\left(\partial_{t}^{2}+P\right) v_{j}=\varphi(t-j)\left[P, \phi_{3 R}\right] u-\left[\partial_{t}^{2}, \varphi(t-j)\right] \phi_{3 R} u+\varphi(t-j) F \\
v_{j}(0, \cdot)=\partial_{t} v_{j}(0, \cdot)=0
\end{array}\right.
$$

while $v_{0}=v-\sum_{j=1}^{\infty} v_{j}$ solves

$$
\left\{\begin{array}{l}
\left(\partial_{t}^{2}+P\right) v_{0}=\tilde{\varphi}\left[P, \phi_{3 R}\right] u-\left[\partial_{t}^{2}, \tilde{\varphi}\right] \phi_{3 R} u+\tilde{\varphi} F \\
\left.v_{0}\right|_{t=0}=u_{0},\left.\partial_{t} v_{0}\right|_{t=0}=u_{1}
\end{array}\right.
$$

if $\tilde{\varphi}=1-\sum_{j=1}^{\infty} \varphi(t-j)$ if $t \geq 0$ and 0 otherwise. 
If we then let $F_{j}=\left(\partial_{t}^{2}+P\right) v_{j}$ be the forcing term for $v_{j}, j=0,1,2, \ldots$, then, by (5.13), we have that

$$
\sum_{j=0}^{\infty}\left\|F_{j}\right\|_{L_{t}^{2} \dot{H}^{s-1}}^{2} \lesssim\left\|u_{0}\right\|_{\dot{H}^{s}}^{2}+\left\|u_{1}\right\|_{\dot{H}^{s-1}}^{2}+\|F\|_{L_{t}^{2} \dot{H}^{s-1}}^{2} .
$$

By the local Strichartz estimates (5.11) and Duhamel's formula, we get for $j=$ $1,2, \ldots$

$$
\left\|v_{j}\right\|_{L_{t}^{q} L_{x}^{r}} \lesssim \int_{0}^{\infty}\left\|F_{j}(s, \cdot)\right\|_{\dot{H}^{s-1}} d s \lesssim\left\|F_{j}\right\|_{L_{t}^{2} \dot{H}^{s-1}},
$$

using Schwarz's inequality and the support properties of the $F_{j}$ in the last step. Similarly,

$$
\left\|v_{0}\right\|_{L_{t}^{q} L_{x}^{r}} \lesssim\left\|u_{0}\right\|_{\dot{H}^{s}}+\left\|u_{1}\right\|_{H^{s-1}}+\left\|F_{0}\right\|_{L_{t}^{2} \dot{H}^{s-1}}
$$

Since $q, r \geq 2$, we have

$$
\|v\|_{L_{t}^{q} L_{x}^{r}}^{2} \lesssim \sum_{j=0}^{\infty}\left\|v_{j}\right\|_{L_{t}^{q} L_{x}^{r}}^{2}
$$

and so we get

$$
\|v\|_{L_{t}^{q} L_{x}^{r}}^{2} \lesssim\left\|u_{0}\right\|_{\dot{H}^{s}}^{2}+\|g\|_{\dot{H}^{s-1}}^{2}+\|F\|_{L_{t}^{2} \dot{H}^{s-1}}^{2}
$$

as desired, which finishes the proof.

End of Proof of Theorem 1.4; Recall that we are assuming that $\left(\partial_{t}^{2}+P\right) u=0$. By Proposition 5.5, we may also assume that the initial data for $u$ vanishes when $|x|<3 R / 2$. We then fix $\beta \in C_{c}^{\infty}\left(\mathbb{R}^{n}\right)$ satisfying $\beta(x)=1,|x| \leq R$ and $\beta(x)=0$, $|x|>3 R / 2$ and write

$$
u=\tilde{u}-v=(1-\beta) \tilde{u}+(\beta \tilde{u}-v),
$$

where $\tilde{u}$ solves the Cauchy problem for $\left(\partial_{t}^{2}+\tilde{P}\right) \tilde{u}=0$ with initial data $\left(u_{0}, u_{1}\right)$. By the global Strichartz estimate (5.8), we can restrict our attention to $w=\beta \tilde{u}-v$. But

$$
\left(\partial_{t}^{2}+P\right) w=[P, \beta] \tilde{u} \equiv G
$$

is supported in $R<|x|<2 R$, and satisfies

$$
\int_{0}^{\infty}\|G(t, \cdot)\|_{\dot{H}^{s-1}}^{2} d t \lesssim\left\|u_{0}\right\|_{\dot{H}^{s}}^{2}+\left\|u_{1}\right\|_{\dot{H}^{s-1}}^{2}
$$

by Lemma 2.6. Note also that $w$ has vanishing initial data. Therefore, since Proposition 5.5 tells us that $\|w\|_{L_{t}^{q} L_{x}^{r}}^{2}$ is dominated by the left side of (5.15), the proof is complete.

\section{Weighted Strichartz Estimates}

Recall Lemma 2.1 and Remark 2.1, we have the following estimates

$$
\begin{aligned}
& \left\|\langle x\rangle^{-1 / 2-\epsilon} e^{i t P^{1 / 2}} f\right\|_{L_{t}^{2} L_{x}^{2}} \lesssim\|f\|_{L_{x}^{2}} \\
& \left\|\langle x\rangle^{-3 / 2-\epsilon} e^{i t P^{1 / 2}} f\right\|_{L_{t}^{2} L_{x}^{2}} \lesssim\|f\|_{\dot{H}_{x}^{1}}
\end{aligned}
$$

By interpolation, we get

$$
\left\||x|^{-\alpha-\epsilon} e^{i t P^{1 / 2}} f\right\|_{L_{t}^{2} L_{|x| \geq R}^{2}} \lesssim\left\|\langle x\rangle^{-\alpha-\epsilon} e^{i t P^{1 / 2}} f\right\|_{L_{t}^{2} L_{x}^{2}} \lesssim\|f\|_{\dot{H}_{x}^{\alpha-1 / 2}}
$$

for any $\alpha \in[1 / 2,3 / 2], \epsilon>0$ and $d \geq 3$. 
Recall that Fang and Wang obtained the following Sobolev inequalities with angular regularity (Corollary 1.2 in [6])

$$
\left\||x|^{\frac{d}{2}-\alpha} f(x)\right\|_{L_{|x|}^{\infty} L_{\omega}^{2+\eta}} \lesssim\left\||x|^{\frac{d}{2}-\alpha} f(x)\right\|_{L_{|x|}^{\infty} H_{\omega}^{\alpha-\frac{1}{2}}} \lesssim\|f\|_{\dot{H}_{x}^{\alpha}}
$$

for $\alpha \in(1 / 2, d / 2)$ and some $\eta>0$. Then by (2.8) in Lemma 2.4, we have

$$
\left\||x|^{\frac{d}{2}-\alpha} e^{i t P^{1 / 2}} f(x)\right\|_{L_{t,|x|}^{\infty} L_{\omega}^{2+\eta}} \lesssim\left\|e^{i t P^{1 / 2}} f(x)\right\|_{L_{t}^{\infty} \dot{H}_{x}^{\alpha}} \lesssim\|f\|_{\dot{H}_{x}^{\alpha}}
$$

for $\alpha \in(1 / 2,1]$ and some $\eta>0$.

If we interpolate between (6.1) and (6.3) we conclude that,

$$
\left\||x|^{\frac{d}{2}-\frac{d+1}{q}-s-\epsilon} e^{i t P^{1 / 2}} f(x)\right\|_{L_{t,|x| \geq R}^{q} L_{\omega}^{2+\eta}}+\left\|\langle x\rangle^{-\frac{1}{2}-s-\epsilon} e^{i t P^{1 / 2}} f(x)\right\|_{L_{t, x}^{2}} \lesssim\|f\|_{\dot{H}_{x}^{s}}
$$

for any $\epsilon>0,2<q \leq \infty$ and $s \in(1 / 2-1 / q, 1]$ with $\eta>0$ small enough. This implies Theorem 1.6 by Duhamel's formula and observation (3.2).

Using the weighted Strichartz estimates, we shall prove the Strauss conjecture in our setting, by adapting the arguments in Hidano-Metcalfe-Smith-Sogge-Zhou 9 .

To this end, we define $X=X_{s, \epsilon, q}\left(\mathbb{R}^{d}\right)$ to be the space with norm defined by

$$
\|h\|_{X_{s, \epsilon, q}}=\|h\|_{L^{q_{s}}(|x| \leq R)}+\|\||x|^{\frac{d}{2}-\frac{d+1}{q}-s-\epsilon} h \|_{L_{|x|}^{q} L_{\omega}^{2+\eta}(|x| \geq R)},
$$

if $d\left(\frac{1}{2}-\frac{1}{q_{s}}\right)=s$. Note that we have the embedding $\dot{H}^{s} \subset X_{s, 0, \infty}$ for $s \in(1 / 2,1]$ by Sobolev embedding and (6.3). By duality, we have

$$
X_{1-s, 0, \infty}^{\prime} \subset \dot{H}^{s-1} \text { for } s \in[0,1 / 2) .
$$

We also denote the space $Y_{s, \epsilon}\left(\mathbb{R}^{d}\right)$ with norm

$$
\|h\|_{Y_{s, \epsilon}}=\left\|\langle x\rangle^{-\frac{1}{2}-s-\epsilon} h\right\|_{L_{x}^{2}} .
$$

Note that by Remark 2.1, duality and interpolation and the homogeneous estimate (6.1), we have

$$
\|u\|_{L_{t}^{2} Y_{s, \epsilon}}+\|u\|_{L_{t}^{\infty} \dot{H}^{s}}+\left\|\partial_{t} u\right\|_{L_{t}^{\infty} \dot{H}^{s-1}} \lesssim\left\|u_{0}\right\|_{\dot{H}^{s}}+\left\|u_{1}\right\|_{\dot{H}_{x}^{s-1}}+\|F\|_{L_{t}^{2} Y_{1-s, \epsilon}^{\prime}}
$$

for the solutions to the linear wave equation (5.1) and $s \in[0,1]$.

Then by (2.14) and energy estimate, if $\left(\partial_{t}^{2}+P\right) u=0$, we have

$$
\|\phi u\|_{L_{t}^{p} \dot{H}^{s}} \lesssim\|\phi u\|_{L_{t}^{\infty} \dot{H}^{s}}+\|\phi u\|_{L_{t}^{2} \dot{H}^{s}} \lesssim\left\|u_{0}\right\|_{\dot{H}^{s}}+\left\|u_{1}\right\|_{\dot{H}_{x}^{s-1}},
$$

for any $\phi \in C_{c}^{\infty}, s \in[0,1]$ and $p \geq 2$. Thus by (6.7) and Christ-Kiselev lemma, we have

$$
\|\phi u\|_{L_{t}^{p} \dot{H}^{s}} \lesssim\left\|u_{0}\right\|_{\dot{H}^{s}}+\left\|u_{1}\right\|_{\dot{H}_{x}^{s-1}}+\|F\|_{L_{t}^{2} Y_{1-s, \epsilon}^{\prime}}
$$

for the solutions of the linear wave equation (5.1), $s \in[0,1]$ and $p>2$.

In conclusion, by (6.3), (6.7) and (6.8), we have

$$
\|u\|_{L_{t}^{\infty} \dot{H}^{s} \cap L_{t}^{p} X_{s, \epsilon, p} \cap L_{t}^{2} Y_{s, \epsilon}}+\left\|\partial_{t} u\right\|_{L_{t}^{\infty} \dot{H}^{s-1}} \lesssim\left\|u_{0}\right\|_{\dot{H}^{s}}+\left\|u_{1}\right\|_{\dot{H}_{x}^{s-1}}+\|F\|_{L_{t}^{2} Y_{1-s, \epsilon}^{\prime}}
$$

for the solutions to the linear wave equation (5.1), if $p>2, s \in(1 / 2-1 / p, 1]$. Here, it will be useful to note that if $d=3,4, p>p_{c}$, i.e. $\frac{d}{2}-\frac{2}{p-1}>\frac{1}{2}-\frac{1}{p}$ and $p>1$, we can choose $\epsilon>0$, so that $s=\frac{d}{2}-\frac{2}{p-1}-\frac{p \epsilon}{p-1} \in(1 / 2-1 / p, 1 / 2)$ and

$$
p\left(\frac{d}{2}-\frac{d+1}{p}-s-\epsilon\right)=-\left(\frac{d}{2}-(1-s)\right)=-s-\frac{d-2}{2} .
$$


Now we want to prove the higher order estimates for (6.9). We claim that if $g^{i j}(x)=h(|x|) \delta^{i j}$,

$$
\begin{aligned}
\sum_{|\alpha|+|\beta| \leq 1}\left(\left\|\partial_{x}^{\alpha} \Omega^{\beta} u\right\|_{L_{t}^{\infty} \dot{H}^{s} \cap L_{t}^{p} X_{s, \epsilon, p} \cap L_{t}^{2} Y_{s, \epsilon}}+\left\|\partial_{t} \partial_{x}^{\alpha} \Omega^{\beta} u\right\|_{L_{t}^{\infty} \dot{H}^{s-1}}\right) & \lesssim \\
\sum_{|\alpha|+|\beta| \leq 1}\left(\left\|\partial_{x}^{\alpha} \Omega^{\beta} u_{0}\right\|_{\dot{H}^{s}}+\left\|\partial_{x}^{\alpha} \Omega^{\beta} u_{1}\right\|_{\dot{H}_{x}^{s-1}}\right) &
\end{aligned}
$$

for the solutions to the linear wave equation (5.1) with $F=0$, if $\rho>0, p>2$, $s \in(1 / 2-1 / p, 1]$. As in the proof of (6.9), we need only to prove the higher order version of (6.3), (6.7) and (6.8).

We first estimate $L_{t}^{2} Y_{s, \epsilon}$ part. By (6.7), (2.4) (2.5) in Lemma2.3 and (2.8) (2.10) in Lemma 2.4, for any $s \in(1 / 2-1 / p, 1]$,

$$
\begin{aligned}
\sum_{|\alpha| \leq 1}\left\|\partial_{x}^{\alpha} u\right\|_{L_{t}^{2} Y_{s, \epsilon}} & \lesssim \sum_{|\alpha| \leq 1}\left\|\tilde{\partial}_{x}^{\alpha} u\right\|_{L_{t}^{2} Y_{s, \epsilon}} \\
& \lesssim \sum_{j \leq 1}\left\|P^{j / 2} u\right\|_{L_{t}^{2} Y_{s, \epsilon / 2}} \\
& \lesssim \sum_{j \leq 1}\left(\left\|P^{j / 2} u_{0}\right\|_{\dot{H}^{s}}+\left\|P^{j / 2} u_{1}\right\|_{\dot{H}_{x}^{s-1}}\right) \\
& \lesssim \sum_{j \leq 1}\left(\left\|P^{(j+s) / 2} u_{0}\right\|_{L_{x}^{2}}+\left\|P^{(j+s-1) / 2} u_{1}\right\|_{L_{x}^{2}}\right) \\
& \lesssim \sum_{|\alpha| \leq 1}\left(\left\|\partial_{x}^{\alpha} u_{0}\right\|_{\dot{H}^{s}}+\left\|\partial_{x}^{\alpha} u_{1}\right\|_{\dot{H}_{x}^{s-1}}\right)
\end{aligned}
$$

Note that since $g^{i j}(x)=h(|x|) \delta^{i j},\left[P, \Omega^{i j}\right]=0$. Thus

$$
\sum_{|\alpha|=1}\left\|\Omega^{\alpha} u\right\|_{L_{t}^{2} Y_{s, \epsilon}} \lesssim \sum_{|\alpha|=1}\left(\left\|\Omega^{\alpha} u_{0}\right\|_{\dot{H}^{s}}+\left\|\Omega^{\alpha} u_{1}\right\|_{\dot{H}_{x}^{s-1}}\right)
$$

In conclusion, we have for $\rho>0$,

$$
\sum_{|\alpha|+|\beta| \leq 1}\left\|\partial_{x}^{\alpha} \Omega^{\beta} u\right\|_{L_{t}^{2} Y_{s, \epsilon}} \lesssim \sum_{|\alpha|+|\beta| \leq 1}\left(\left\|\partial_{x}^{\alpha} \Omega^{\beta} u_{0}\right\|_{\dot{H}^{s}}+\left\|\partial_{x}^{\alpha} \Omega^{\beta} u_{1}\right\|_{\dot{H}_{x}^{s-1}}\right) .
$$

We turn to the proof of the higher order estimate for (6.3). Note that we assume $g^{i j}(x)=h(|x|) \delta^{i j}$, then $P=-h \Delta+r_{1} \partial+r_{2}$, and hence by Hardy inequality and (2.8) in Lemma 2.4

$$
\|\partial u\|_{\dot{H}^{1}} \lesssim\|\Delta u\|_{L^{2}} \lesssim\|P u\|_{L^{2}}+\|\partial u\|_{L^{2}}+\left\|r_{2} u\right\|_{L^{2}} \lesssim\left\|P^{1 / 2} u\right\|_{H^{1}} .
$$

If we interpolate with (2.9), we get that for $s \in[0,1]$,

$$
\|\partial u\|_{\dot{H}^{s}} \lesssim\left\|P^{1 / 2} u\right\|_{H^{s}} .
$$


By (6.3), (6.13), (2.8) and (2.10) in Lemma 2.4) if $\left(\partial_{t}^{2}+P\right) u=0$ and $s \in(1 / 2,1]$, we have the energy estimate,

$$
\begin{aligned}
\sum_{|\alpha| \leq 1}\left\|\partial_{x}^{\alpha} u\right\|_{L_{t}^{\infty} \dot{H}^{s}} & \lesssim \sum_{j \leq 1}\left\|P^{j / 2} u\right\|_{L_{t}^{\infty} \dot{H}^{s}} \\
& \lesssim \sum_{j \leq 1}\left(\left\|P^{j / 2} u(0)\right\|_{\dot{H}^{s}}+\left\|P^{j / 2} \partial_{t} u(0)\right\|_{\dot{H}^{s-1}}\right) \\
& \lesssim \sum_{|\alpha| \leq 1}\left(\left\|\tilde{\partial}_{x}^{\alpha} u(0)\right\|_{\dot{H}_{x}^{s}}+\left\|\tilde{\partial}_{x}^{\alpha} \partial_{t} u(0)\right\|_{\dot{H}_{x}^{s-1}}\right) \\
& \lesssim \sum_{|\alpha| \leq 1}\left(\left\|\partial_{x}^{\alpha} u(0)\right\|_{\dot{H}_{x}^{s}}+\left\|\partial_{x}^{\alpha} \partial_{t} u(0)\right\|_{\dot{H}_{x}^{s-1}}\right)
\end{aligned}
$$

and

$$
\begin{aligned}
\sum_{|\alpha| \leq 1}\left\||x|^{\frac{d}{2}-s} \tilde{\partial}_{x}^{\alpha} u\right\|_{L_{[0, T],|x|}^{\infty} L_{\omega}^{2+\eta}} & \lesssim \sum_{|\alpha| \leq 1}\left(\left\|\tilde{\partial}_{x}^{\alpha} u(0)\right\|_{\dot{H}_{x}^{s}}+\left\|\tilde{\partial}_{x}^{\alpha} \partial_{t} u(0)\right\|_{\dot{H}_{x}^{s-1}}\right)+\left\|\left[P, \tilde{\partial}_{x}\right] u\right\|_{L_{T}^{1} \dot{H}_{x}^{s-1}} \\
& \lesssim \sum_{|\alpha| \leq 1}\left(\left\|\partial_{x}^{\alpha} u(0)\right\|_{\dot{H}_{x}^{s}}+\left\|\partial_{x}^{\alpha} \partial_{t} u(0)\right\|_{\dot{H}_{x}^{s-1}}\right)+\sum_{1 \leq|\beta| \leq 2}\left\|\tilde{\partial}_{x}^{\beta} u\right\|_{L_{T}^{1} \dot{H}_{x}^{s-1}} \\
& \lesssim \sum_{|\alpha| \leq 1}\left(\left\|\partial_{x}^{\alpha} u(0)\right\|_{\dot{H}_{x}^{s}}+\left\|\partial_{x}^{\alpha} \partial_{t} u(0)\right\|_{\dot{H}_{x}^{s-1}}\right)+\sum_{|\beta| \leq 1}\left\|\tilde{\partial}_{x}^{\beta} u\right\|_{L_{T}^{1} \dot{H}_{x}^{s}} \\
& \lesssim(1+T) \sum_{|\alpha| \leq 1}\left(\left\|\partial_{x}^{\alpha} u(0)\right\|_{\dot{H}_{x}^{s}}+\left\|\partial_{x}^{\alpha} \partial_{t} u(0)\right\|_{\dot{H}_{x}^{s-1}}\right) .
\end{aligned}
$$

Thus for any $k \in \mathbb{Z}$,

$$
\begin{aligned}
\sum_{|\alpha| \leq 1}\left\||x|^{\frac{d}{2}-s} \partial_{x}^{\alpha} u(t)\right\|_{L_{t \in[k, k+1],|x|}^{\infty} L_{\omega}^{2+\eta}} & \lesssim \sum_{|\alpha| \leq 1}\left\||x|^{\frac{d}{2}-s} \tilde{\partial}_{x}^{\alpha} u\right\|_{L_{t \in[k, k+1],|x|}^{\infty} L_{\omega}^{2+\eta}} \\
& \lesssim \sum_{|\alpha| \leq 1}\left(\left\|\partial_{x}^{\alpha} u(k)\right\|_{\dot{H}_{x}^{s}}+\left\|\partial_{x}^{\alpha} \partial_{t} u(k)\right\|_{\dot{H}_{x}^{s-1}}\right) \\
& \lesssim \sum_{|\alpha| \leq 1}\left(\left\|\partial_{x}^{\alpha} u(0)\right\|_{\dot{H}_{x}^{s}}+\left\|\partial_{x}^{\alpha} \partial_{t} u(0)\right\|_{\dot{H}_{x}^{s-1}}\right)
\end{aligned}
$$

Hence we get the higher order version of (6.3) if we combine the commutativity of $P$ and $\Omega$,

$$
\sum_{|\alpha|+|\beta| \leq 1}\left\||x|^{\frac{d}{2}-s} \partial_{x}^{\alpha} \Omega^{\beta} u(t)\right\|_{L_{t,|x|}^{\infty} L_{\omega}^{2+\eta}} \lesssim \sum_{|\alpha|+|\beta| \leq 1}\left(\left\|\partial_{x}^{\alpha} \Omega^{\beta} u_{0}\right\|_{\dot{H}_{x}^{s}}+\left\|\partial_{x}^{\alpha} \Omega^{\beta} u_{1}\right\|_{\dot{H}_{x}^{s-1}}\right)
$$

for the solution to the linear wave equation (5.1) with $F=0$, and $s \in(1 / 2,1]$.

We need only to prove the higher order version of (6.8) now.

By (3.6), we know that if $\left(\partial_{t}^{2}+P\right) u=0$ and $\rho>0$,

$$
\begin{gathered}
\left\|\langle x\rangle^{-1 / 2-\epsilon} \partial_{x} u\right\|_{L_{t}^{2} \dot{H}^{1}} \lesssim \sum_{|\alpha|=2}\left\|\langle x\rangle^{-1 / 2-\epsilon} \partial_{x}^{\alpha} u\right\|_{L_{t, x}^{2}}+\left\|\langle x\rangle^{-3 / 2-\epsilon} \partial_{x} u\right\|_{L_{t, x}^{2}} \lesssim\left\|\partial_{x} u(0)\right\|_{H_{x}^{1}}+\left\|\partial_{t} u(0)\right\|_{H_{x}^{1}}, \\
\left\|\langle x\rangle^{-1 / 2-\epsilon} \partial_{x} u\right\|_{L_{t, x}^{2}} \lesssim\left\|\partial_{x} u(0)\right\|_{L_{x}^{2}}+\left\|\partial_{t} u(0)\right\|_{L_{x}^{2}},
\end{gathered}
$$


Thus for $s \in[0,1]$,

$$
\left\|\phi \partial_{x} u\right\|_{L_{t}^{2} \dot{H}^{s}} \lesssim\|\partial u(0)\|_{H_{x}^{s}}+\left\|\partial_{t} u(0)\right\|_{H_{x}^{s}} \lesssim \sum_{|\alpha| \leq 1}\left(\left\|\partial_{x}^{\alpha} u(0)\right\|_{\dot{H}_{x}^{s}}+\left\|\partial_{x}^{\alpha} \partial_{t} u(0)\right\|_{\dot{H}_{x}^{s-1}}\right) .
$$

Combining the energy estimates, we know that

$$
\sum_{|\alpha|+|\beta| \leq 1}\left\|\phi \partial_{x}^{\alpha} \Omega^{\beta} u\right\|_{L_{t}^{p} \dot{H}^{s}} \lesssim \sum_{|\alpha|+|\beta| \leq 1}\left(\left\|\partial_{x}^{\alpha} \Omega^{\beta} u_{0}\right\|_{\dot{H}^{s}}+\left\|\partial_{x}^{\alpha} \Omega^{\beta} u_{1}\right\|_{\dot{H}_{x}^{s-1}}\right)
$$

for the solutions to the linear wave equation (5.1) with $F=0, s \in[0,1]$ and $p>2$. This completes the proof of (6.11).

To conclude this section, let us point out that a similar estimate of (6.11) holds for the solution to (1.5), as in the end of Section 3. Precisely, if $g^{i j}(x)=h(|x|) \delta^{i j}$, we have

$$
\begin{gathered}
\sum_{|\alpha|+|\beta| \leq 1}\left(\left\|\partial_{x}^{\alpha} \Omega^{\beta} u\right\|_{L_{t}^{\infty} \dot{H}^{s} \cap L_{t}^{p} X_{s, \epsilon, p} \cap L_{t}^{2} Y_{s, \epsilon}}+\left\|\partial_{t} \partial_{x}^{\alpha} \Omega^{\beta} u\right\|_{L_{t}^{\infty} \dot{H}^{s-1}}\right) \\
\sum_{|\alpha|+|\beta| \leq 1}\left(\left\|\partial_{x}^{\alpha} \Omega^{\beta} u_{0}\right\|_{\dot{H}^{s}}+\left\|\partial_{x}^{\alpha} \Omega^{\beta} u_{1}\right\|_{\dot{H}_{x}^{s-1}}\right)
\end{gathered}
$$

for the solutions to the linear wave equation (1.5) with $F=0$, if $\rho>0, p>2$, $s \in(1 / 2-1 / p, 1]$.

\section{Strauss Conjecture}

In this section, we prove the Strauss conjecture in the setting where $g^{i j}=$ $h(|x|) \delta^{i j}, p>p_{c}=1+\sqrt{2}$ and $d=3$, i.e., Theorem 1.5 .

By (6.16), (6.6) and Duhamel's formula, we have

$$
\begin{gathered}
\sum_{|\alpha|+|\beta| \leq 1}\left(\left\|\partial_{x}^{\alpha} \Omega^{\beta} u\right\|_{L_{t}^{\infty} \dot{H}^{s} \cap L_{t}^{p} X_{s, \epsilon, p}}+\left\|\partial_{t} \partial_{x}^{\alpha} \Omega^{\beta} u\right\|_{L_{t}^{\infty} \dot{H}^{s-1}}\right) \\
\lesssim \quad \sum_{|\alpha|+|\beta| \leq 1}\left(\left\|\partial_{x}^{\alpha} \Omega^{\beta} u_{0}\right\|_{\dot{H}^{s}}+\left\|\partial_{x}^{\alpha} \Omega^{\beta} u_{1}\right\|_{\dot{H}_{x}^{s-1}}+\left\|\partial_{x}^{\alpha} \Omega^{\beta} F\right\|_{L_{t}^{1} \dot{H}^{s-1}}\right) \\
\lesssim \sum_{|\alpha|+|\beta| \leq 1}\left(\left\|\partial_{x}^{\alpha} \Omega^{\beta} u_{0}\right\|_{\dot{H}^{s}}+\left\|\partial_{x}^{\alpha} \Omega^{\beta} u_{1}\right\|_{\dot{H}_{x}^{s-1}}+\left\|\partial_{x}^{\alpha} \Omega^{\beta} F\right\|_{L_{t}^{1} X_{1-s, 0, \infty}^{\prime}}\right)
\end{gathered}
$$

for the solutions to the linear wave equation (1.5), if $\rho>0, p>2, s \in(1 / 2-$ $1 / p, 1 / 2)$.

Let us now see how we can use these estimates to prove Theorem 1.5. We assume Cauchy data $\left(u_{0}, u_{1}\right)$ satsifying the smallness condition (1.12), and let $u^{(0)}$ solve the Cauchy problem (1.9) with $F=0$. We iteratively define $u^{(k)}$, for $k \geq 1$, by solving

$$
\left\{\begin{array}{l}
\left(\partial_{t}^{2}-\Delta_{\mathfrak{g}}\right) u^{(k)}(t, x)=F_{p}\left(u^{(k-1)}(t, x)\right), \quad(t, x) \in \mathbb{R}_{+} \times \Omega \\
u(0, \cdot)=u_{0}, \quad \partial_{t} u(0, \cdot)=u_{1} .
\end{array}\right.
$$

Our aim is to show that if the constant $\delta>0$ in (1.12) is small enough, then so is

$$
M_{k}=\sum_{|\alpha| \leq 1}\left(\left\|Y^{\alpha} u^{(k)}\right\|_{L_{t}^{\infty} \dot{H}^{s} \cap L_{t}^{p} X_{s, \epsilon, p}}+\left\|\partial_{t} Y^{\alpha} u^{(k)}\right\|_{L_{t}^{\infty} \dot{H}^{s-1}}\right)
$$

for every $k=0,1,2, \ldots$ 
For $k=0$, it follows by (7.1) that $M_{0} \leq C_{0} \delta$, with $C_{0}$ a fixed constant. More generally, for some $\eta_{1} \in(0,1)$, (17.1) implies that

$$
\begin{aligned}
M_{k} \leq C_{0} \delta+C_{0} \sum_{|\alpha| \leq 1}( & \left\||x|^{-\frac{d}{2}+1-s} Y^{\alpha} F_{p}\left(u^{(k-1)}\right)\right\|_{L_{t}^{1} L_{|x|}^{1} L_{\omega}^{2-\eta_{1}}\left(\mathbb{R}_{+} \times\{|x| \geq R\}\right)} \\
& \left.+\left\|Y^{\alpha} F_{p}\left(u^{(k-1)}\right)\right\|_{L_{t}^{1} L_{x}^{q_{1}^{\prime}-s}\left(\mathbb{R}_{+} \times\{x \in \Omega:|x| \leq R\}\right)}\right) .
\end{aligned}
$$

Note that our assumption (1.10) on the nonlinear term $F_{p}$ implies that for small $v$

$$
\sum_{|\alpha| \leq 1}\left|Y^{\alpha} F_{p}(v)\right| \lesssim|v|^{p-1} \sum_{|\alpha| \leq 1}\left|Y^{\alpha} v\right|
$$

Since the collection $Y$ contains vectors spanning the tangent space to $S^{2}$, by Sobolev embedding we have for any $q \leq \infty$ and $\eta_{2} \in(0,1)$

$$
\|v(r \cdot)\|_{L_{\omega}^{q}} \lesssim \sum_{|\alpha| \leq 1}\left\|Y^{\alpha} v(r \cdot)\right\|_{L_{\omega}^{2+\eta_{2}}} .
$$

Consequently, for fixed $t, r>0$

$$
\sum_{|\alpha| \leq 1}\left\|Y^{\alpha} F_{p}\left(u^{(k-1)}(t, r \cdot)\right)\right\|_{L_{\omega}^{2-\eta_{1}}} \lesssim \sum_{|\alpha| \leq 1}\left\|Y^{\alpha} u^{(k-1)}(t, r \cdot)\right\|_{L_{\omega}^{2+\eta_{2}}}^{p} .
$$

By (6.10), the first summand in the right side of (7.2) is dominated by $C_{1} M_{k-1}^{p}$.

We next observe that, for each fixed $t$, we have

$\sum_{|\alpha| \leq 1}\left\|Y^{\alpha} F_{p}\left(u^{(k-1)}(t, \cdot)\right)\right\|_{L^{q_{1-s}^{\prime}(x:|x| \leq R)}} \lesssim \sum_{|\alpha| \leq 1}\|u\|_{L^{q}(x:|x| \leq R)}^{p-1}\left\|Y^{\alpha} u^{(k-1)}(t, \cdot)\right\|_{L^{q_{s}}(x:|x| \leq R)}$,

where

$$
\frac{1}{q}=\frac{1}{p-1}\left(\frac{1}{q_{1-s}^{\prime}}-\frac{1}{q_{s}}\right)=\frac{1}{3(p-1)} .
$$

It follows by Sobolev embedding on $\{x:|x| \leq R\}$ that

$$
\|v\|_{L^{q}(x:|x| \leq R)} \lesssim \sum_{|\alpha| \leq 1}\left\|Y^{\alpha} v\right\|_{L^{q_{s}}(x:|x| \leq R)},
$$

since $s \in\left(\frac{1}{2}-\frac{1}{p}, \frac{1}{2}\right) \subset\left[\frac{1}{2}-\frac{1}{p-1}, \frac{3}{2}-\frac{1}{p-1}\right]$, i.e. $\frac{3}{q}=\frac{1}{p-1} \in\left[-1+\frac{3}{q_{s}}, \frac{3}{q_{s}}\right]$.

The second summand in the right side of (7.2) is thus also dominated by $C_{1} M_{k-1}^{p}$, and we conclude that $M_{k} \leq C_{0} \delta+2 C_{0} C_{1} M_{k-1}^{p}$. For $\delta$ sufficiently small, then

$$
M_{k} \leq 2 C_{0} \delta, \quad k=1,2,3, \ldots
$$

To finish the proof of Theorem 1.5 we need to show that $u^{(k)}$ converges to a solution of the equation (1.9). For this it suffices to show that

$$
A_{k}=\left\|u^{(k)}-u^{(k-1)}\right\|_{L_{t}^{p} X_{s, \epsilon, p}}
$$

tends geometrically to zero as $k \rightarrow \infty$. Since $\left|F_{p}(v)-F_{p}(w)\right| \lesssim|v-w|\left(|v|^{p-1}+\right.$ $\left.|w|^{p-1}\right)$, the proof of (7.3) can be adapted to show that, for small $\delta>0$, there is a uniform constant $C$ so that

$$
A_{k} \leq C A_{k-1}\left(M_{k-1}+M_{k-2}\right)^{p-1}
$$

which, by (7.3), implies that $A_{k} \leq \frac{1}{2} A_{k-1}$ for small $\delta$. Since $A_{1}$ is finite, the claim follows, which finishes the proof of Theorem 1.5 . 


\section{REFERENCES}

[1] H. Bahouri, J. Y. Chemin, Equations dondes quasilineaires et estimations de Strichartz (Quasilinear wave equations and Strichartz estimates), Amer. J. Math. 121 (1999), 13371377.

[2] H. Bahouri, J. Y. Chemin, Equations dondes quasilineaires et effet dispersif (Quasilinear wave equations and dispersive effect), Internat. Math. Res. Notices 1999, No. 21, 1141-1178.

[3] J.-F. Bony, D. Häfner, The semilinear wave equation on asymptotically Euclidean manifolds, Comm. in PDE 35(2010), no. 1, 23-67.

[4] N. Burq, Global Strichartz estimates for nontrapping geometries: about an article by $H$. F. Smith and C. D. Sogge: "Global Strichartz estimates for nontrapping perturbations of the Laplacian", Comm. Partial Differential Equations, 28 (2003), 1675-1683.

[5] M. Christ, A. Kiselev, Maximal Functions Associated to Filtrations, Jour. Func. Anal. 179 (2001), 409-425.

[6] D. Fang, C. Wang, Weighted Strichartz Estimates with Angular Regularity and their Applications, Forum Math., to appear.

[7] V. Georgiev, H. Lindblad, C. D. Sogge, Weighted Strichartz estimates and global existence for semilinear wave equations, American Journal of Mathematics, 119 (1997), 1291-1319.

[8] R. T. Glassey, Existence in the large for $\square u=F(u)$ in two dimensions, Math. Z. 178 (1981), $233-261$.

[9] K. Hidano, J. Metcalfe, H. F. Smith, C. D. Sogge, Y. Zhou, On Abstract Strichartz Estimates and the Strauss Conjecture for Nontrapping Obstacles, Trans. Amer. Math. Soc., 362 (2010), 2789-2809.

[10] F. John, Blow-up for quasilinear wave equations in three space dimensions, Comm. Pure Appl. Math. 34 (1981), no. 1, 29-51.

[11] F. John, S. Klainerman, Almost global existence to nonlinear wave equations in three space dimensions, Comm. Pure Appl. Math. 37 (1984), no. 4, 443-455.

[12] J.-L. Journé, A. Soffer, C. D. Sogge Decay estimates for Schrödinger equations Comm. Pure Appl. Math. 44 (1991), 573-604.

[13] L. V. Kapitanski, Norm estimates in Besov and Lizorkin-Triebel spaces for the solutions of second-order linear hyperbolic equations, J. Soviet Math. 56 (1991), 2348-2389.

[14] M. Keel, H. Smith, C. D. Sogge, Almost global existence for some semilinear wave equations, Dedicated to the memory of Thomas H. Wolff. J. Anal. Math. 87 (2002), 265-279.

[15] M. Keel, H. F. Smith, C. D. Sogge, Almost global existence for quasilinear wave equations in three space dimensions, J. Amer. Math. Soc. 17 (2004), no. 1, 109-153 (electronic).

[16] M. Keel, T. Tao, Endpoint Strichartz Estimates, Amer. J. Math. 120 (1998), 955-980.

[17] C. E. Kenig, G. Ponce, and L. Vega, On the Zakharov and Zakharov-Schulman systems. J. Funct. Anal. 127 (1995), 204-234.

[18] S. Klainerman, Uniform decay estimates and the Lorentz invariance of the classical wave equation, Comm. Pure Appl. Math. 38 (1985), no. 3, 321-332.

[19] S. Klainerman, G. Ponce, Global, small amplitude solutions to nonlinear evolution equations, Comm. Pure Appl. Math. 36 (1983), no. 1, 133-141.

[20] H. Lindblad, C. D. Sogge, On Existence and Scattering with Minimal Regularity for Semilinear Wave Equations, J. Func. Anal. 130 (1995), 357-426.

[21] H. Lindblad and C. D. Sogge, Long-time existence for small amplitude semilinear wave equations, Amer. J. Math. 118 (1996), 1047-1135.

[22] J. Metcalfe, Global Strichartz estimates for solutions to the wave equation exterior to a convex obstacle, Trans. Amer. Math. Soc. 356 (2004), 4839-4855.

[23] J. Metcalfe, C. Sogge, Long-time existence of quasilinear wave equations exterior to starshaped obstacles via energy methods, SIAM J. Math. Anal. 38 (2006), no. 1, 188-209 (electronic).

[24] J. Metcalfe, D. Tataru, Global parametrices and dispersive estimates for variable coefficient wave equations, arXiv:0707.1191.

[25] G. Mockenhaupt, A. Seeger, C. D. Sogge, Local smoothing of Fourier integral operators and Carleson-Sjölin estimates, J. Amer. Math. Soc., 6(1993), no.1, 65-130.

[26] C. S. Morawetz, Time decay for the nonlinear Klein-Gordon equation, Proc. Roy. Soc. A306 (1968), 291-296. 
[27] T. Sideris, Global behavior of solutions to nonlinear wave equations in three dimensions, Comm. Partial Differential Equations 8 (1983), no. 12, 1291-1323.

[28] T. C. Sideris, Nonexistence of global solutions to semilinear wave equations in high dimensions, J. Differential Equations 52 (1984), 378-406.

[29] H. F. Smith, A parametrix construction for wave equations with $C^{1,1}$ coefficients, Ann. Inst. Fourier (Grenoble), 48(1998), no.3, 797-835.

[30] H. F. Smith, C. D. Sogge, Global Strichartz estimates for nontrapping perturbations of the Laplacian, Comm. Partial Differential Equations 25 (2000), no. 11-12, 2171-2183.

[31] C. D. Sogge, Lectures on nonlinear wave equations. Second edition. International Press, Boston, MA, 2008.

[32] G. Staffilani, D. Tataru, Strichartz estimates for a Schrödinger equation with nonsmooth coefficients Comm. Partial Differential Equations 27 (2002), 1337-1372.

[33] W. Strauss, Dispersal of waves vanishing on the boundary of an exterior domain. Comm. Pure Appl. Math. 28 (1975), 265-278.

[34] D. Tataru, Strichartz estimates for operators with nonsmooth coefficients and the nonlinear wave equation, Amer. J. Math., 122(2000), no.2, 349-376.

[35] D. Tataru, Strichartz estimates for second order hyperbolic operators with nonsmooth coefficients. II, Amer. J. Math., 123(2001), no.3, 385-423.

[36] D. Tataru, Strichartz estimates in the hyperbolic space and global existence for the semilinear wave equation, Trans. Amer. Math. Soc. 353 (2001), no. 2, 795-807.

[37] D. Tataru, Strichartz estimates for second order hyperbolic operators with nonsmooth coefficients. III, J. Amer. Math. Soc., 15(2):419-442 (electronic), 2002.

[38] Y. Zhou, Cauchy problem for semilinear wave equations with small data in four space dimensions, J. Partial Differential Equations 8 (1995), 135-144.

Department of Mathematics, Johns Hopkins University, Baltimore, Maryland 21218

E-mail address: sogge@jhu.edu

Department of Mathematics, Johns Hopkins University, Baltimore, Maryland 21218

E-mail address: wangcbo@jhu.edu 